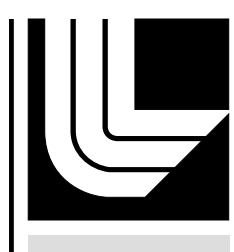

LAW RENCE LIVERMORE N A T IO N A L LABORATORY

SI PC104 Performance Test Report

S. Montelongo

January 20, 2006 
This document was prepared as an account of work sponsored by an agency of the United States Government. Neither the United States Government nor the University of California nor any of their employees, makes any warranty, express or implied, or assumes any legal liability or responsibility for the accuracy, completeness, or usefulness of any information, apparatus, product, or process disclosed, or represents that its use would not infringe privately owned rights. Reference herein to any specific commercial product, process, or service by trade name, trademark, manufacturer, or otherwise, does not necessarily constitute or imply its endorsement, recommendation, or favoring by the United States Government or the University of California. The views and opinions of authors expressed herein do not necessarily state or reflect those of the United States Government or the University of California, and shall not be used for advertising or product endorsement purposes.

This work was performed under the auspices of the U.S. Department of Energy by University of California, Lawrence Livermore National Laboratory under Contract W-7405-Eng-48. 


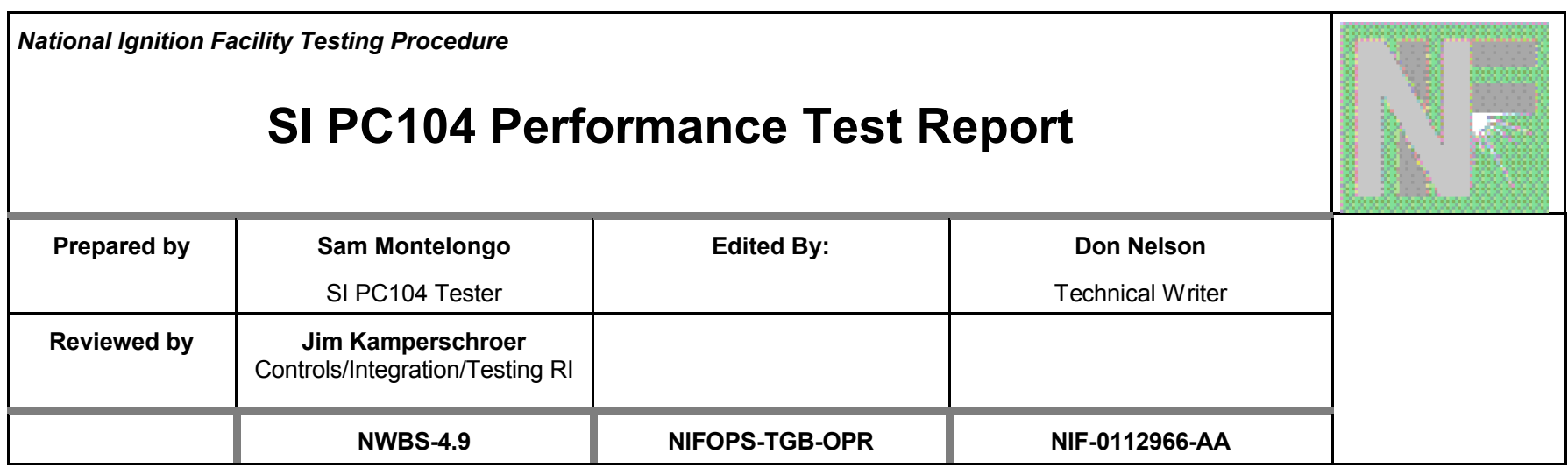

Form 001138-0A

This document describes the procedures carried out during the actual tests. This document is an official record of the actual testing procedures carried out and the subsequent results of those tests. This document will be registered in ECMS.

\subsection{Purpose and Scope of Testing}

1.1 The Spectral Instruments (SI) PC104 systems associated with the SI-1000 CCD camera exhibited intermittent power problems during setup, test and operations which called for further evaluation and testing. The SI PC104 System is the interface between the SI-1000 CCD camera and its associated Diagnostic Controller (DC). As such, the SI PC104 must be a reliable, robust system capable of providing consistent performance in various configurations and operating conditions. This SI PC104 system consists of a stackable set of modules designed to meet the PC104+ Industry Standard. The SI PC104 System consists of a CPU module, SI Camera card, Media converter card, Video card and a I/O module.

1.2 The root cause of power problems was identified as failing solder joints at the LEMO power connector attached to the SI Camera Card. The recommended solution was to provide power to the PC104 system via a PC104+ power supply module configured into the PC104 stack instead of thru the LEMO power connector.

1.3 Test plans (2) were developed to test SI PC104 performance and identify any outstanding issues noted during extended operations. Test Plan 1 included performance and image acquisition tests. Test Plan 2 verified performance after implementing recommendations. Test Plan 2 also included verifying integrity of system files and driver installation after bootup.

1.4 Each test plan was implemented to fully test against each set of problems noted. Test Plan presentations and Test Plan results are attached as appendices.

1.5 Anticipated test results will show successful operation and reliable performance of the SI PC104 system receiving its power via a PC104 power supply module. A SI PC104 Usage 


\section{SI PC104 Performance Test Report}

Recommendation Memo will be sent out to the SI PC104 User Community. Recommendation memo(s) are attached as appendices.

1.6 SI PC104 test plans, results, and recommendations were a result of the PC104 Working Group Team established to address PC104 issues found during design, test, and implementation.

The team consisted of

Sam Montelongo,

Ken Piston,

James Moody,

Jarom Nelson.

With input from: Dennis O'Brien, Joe Kimbrough, and Joe Holder.

-Test Plan 1: Date Testing Begun: June 27, 2005

- Test Plan 2: Date Testing Begun: September 12, 2005

\subsection{Materials and Equipment Required}

2.1 List std. tools and equipment used during tests

\begin{tabular}{|l|l|l|}
\hline \multicolumn{1}{|c|}{ Test or Diagnostic Equipment } & \multicolumn{1}{|c|}{ Part/Model \# } & Qty. \\
\hline Laptop (runs UEC Test code and interacts with the DANTE SI PC104) & Dell & 1 \\
\hline $\begin{array}{l}\text { KONTRON PC104 System (monitors and records PC104 temperature and } \\
\text { buss voltages) }\end{array}$ & Horizon & 1 \\
\hline $\begin{array}{l}\text { Video Monitor (shows debug and system information associated with the } \\
\text { DANTE SI PC104) }\end{array}$ & N/A & 1 \\
\hline SI-1000 CCD camera \#1 (used for testing camera performance) & SN 1000-109 & 1 \\
\hline $\begin{array}{l}\text { SI-1000 CCD camera\#2 (used for comparing camera performance with } \\
\text { camera \#1) }\end{array}$ & SN 1000-106 & 1 \\
\hline SI 1000 Simulator (in place of a SI-1000 CCD camera) & N/A & 1 \\
\hline Tektronix Scope (record PC104 buss voltage waveforms) & Tek-684B & 1 \\
\hline Polyscience Chiller & 5005 & 1 \\
\hline
\end{tabular}

\subsection{Special Test Equipment}




\section{SI PC104 Performance Test Report}

National Ignition Facility Testing Procedure

NIF-\# 0112966-AA

Page 3 of 53

List any nonstandard NIF test equipment and tools needed to perform the test. For each item, include model and part numbers and the quantity needed. Equipment requiring calibration and/or certification should be so indicated.

\begin{tabular}{|l|l|l|l|}
\hline \multicolumn{1}{|c|}{ Test or Diagnostic Equipment } & Part/Model \# & Qty. & \multicolumn{1}{|c|}{$\begin{array}{c}\text { Calibration/ } \\
\text { Certification (YIN) }\end{array}$} \\
\hline $\begin{array}{l}\text { DANTE PC104 System with high voltage capability } \\
\text { disabled }\end{array}$ & N/A & 1 & N \\
\hline $\begin{array}{l}\text { SI PC104 Camera Card \#1 (available to modify per SI } \\
\text { PC104 User Recommendation) }\end{array}$ & $\# 107$ & 1 & N \\
\hline $\begin{array}{l}\text { SI PC104 Camera Card \#2 (available to modify per SI } \\
\text { PC104 User Recommendation for comparison tests) }\end{array}$ & $\# 104$ & 1 & N \\
\hline
\end{tabular}

\subsection{Test Plans}

3.1 Test plans (two total) were developed to test SI PC104 performance and identify any outstanding issues noted during extended operations. Test Plan 1 (One) included performance and image acquisition tests. Test Plan 2 (Two) verified performance after implementing recommendations. Test Plan 2 also included verifying integrity of system files and driver installation after bootup.

3.2 The SI PC104 test system was provided by the DANTE diagnostic. This diagnostic is the most extreme configuration of the PC104 system currently available. The DANTE diagnostic system consists of SI PC104 System stack with additional components added to the PC104 stack support DANTE specific functions.

-SI PC104 System Stack

-DANTE Timing I/O Board

- CCD Buffer Card

3.3 Test Plan 1 (ONE) system configuration, setup and specific set of tests is defined in Appendix A "Test Plan 1 for the SI-1000 PC104 Camera Card".

- The test goals include:

- Implement a continuous cycle image acquisition test on a SI PC104 Camera card configured to receive its power from a PC104+ (Tri- M Engineering HE104+DX) power supply module.

- Identify and fix failure modes of the modified SI PC104 Camera Card when operated over an extended period of time.

- Provide guidelines for using the SI PC104 Camera card in diagnostic configurations 


\begin{tabular}{|c|c|c|}
\hline \multicolumn{2}{|c|}{ SI PC104 Performance Test Report } & 1. \\
\hline National Ignition Facility Testing Procedure & NIF-\# 0112966-AA & Page 4 of 53 \\
\hline
\end{tabular}

3.4 Test Plan 2 (TWO) system configuration, setup and specific set of tests is defined in Appendix C "Test Plan 2 for the SI-1000 PC104 Camera Card". SI PC104. System performance test goals include:

- The test goals include:

- Validate performance of a fully modified (per recommendation) PC104 SI camera card. This test to include testing with the SITASJNIInterface.DLL dated August 9,2005. This code fixes the Exposure/Read issue causing abort of image acquisition.

-Verify operation of Enhanced Write Filter (EWF) on UEC PC104 system. Confirm that power cycling issues are not causing file corruption (i.e. file corruption should be impossible with EWF turned ON)

- Diagnose power cycle and initialization problems on SI PC104 system and provide recommendation for proper procedure.

\subsection{Testing Operations}

4.1 Test Plan 1 (ONE): SI PC104 System configuration changes

- Add PC104+ Power Supply module into SI PC104 stack

- Install modified SI PC104 camera card (SI-1000-107)

1) Remove LEMO power connector

2) Detach input and output legs of the $+3.3 v d c$ on-board regulator.

4.2 Test Plan 2 (TWO): SI PC104 configuration changes

- Install PC104 Power Supply module into SI PC104 stack

-Install modified SI PC104 camera card (SI-1000-107)

1) Remove LEMO power connector

2) Fully attach all pins of the $+3.3 v d c$ on-board regulator

Any operator action(s) required can be listed in the table below

\begin{tabular}{|c|c|c|}
\hline Step & Operator Action Required & Expected Results/Comments \\
& & \\
\hline
\end{tabular}




\section{SI PC104 Performance Test Report}

\begin{tabular}{|c|l|l|}
\hline 1 & $\begin{array}{l}\text { Run tests per Test Plan 1 } \\
\text { using modified SI PC104 } \\
\text { camera card \#1 (LEMO } \\
\text { connector removed, +3.3vdc } \\
\text { regulator detached) and } \\
\text { PC104+ Power Supply } \\
\text { module. }\end{array}$ & $\begin{array}{l}\text { Identify performance issues and run } \\
\text { comparison tests as needed with SI } \\
\text { PC104 camera card \#2 and camera \# } \\
\text { SI-1000-106 }\end{array}$ \\
\hline 2 & $\begin{array}{l}\text { Run tests per Test Plan 2 } \\
\text { using modified SI PC104 } \\
\text { camera card \#1 (LEMO } \\
\text { connector removed, +3.3vdc } \\
\text { regulator attached) and } \\
\text { PC104 Power Supply } \\
\text { module. }\end{array}$ & $\begin{array}{l}\text { Identify performance issues and run } \\
\text { comparison tests as needed with SI } \\
\text { PC104 camera card \#2 and camera \# } \\
\text { Sl-1000-106 }\end{array}$ \\
\hline
\end{tabular}

\subsection{Test results}

5.1 Two Test Plans were implemented over the course of testing. Each test plan was implemented to fully test against each set of problems noted. Test plan results presentations are attached as:

- Appendix B "Test Plan 1 Results for the SI-1000 PC104 Camera Card"

- Appendix D "Test Plan 2 Results for the SI-1000 PC104 Camera Card."

\subsection{Test Plan 1 Results Summary:}

- Data log files associated with these tests are identified in Section 6.3 Data File Locations.

- Continuous cycles of image acquisition showed no image acquisition failures due to power failure or CPU performance.

- Image acquisition failures were noted due to camera Exposure Time query during data read out but did not happen consistently (average about 1 in 10). UEC test logs showed "CANCEL" message during extended image acquisition cycles.

If exposure time is queried during readout, camera may (usually) abort image acquisition. Confirmed by vendor and fixed in newer releases of vendor supplied camera firmware.

LLNL UEC software will be fixed such that Exposure Time queries will not happen during data readout.

- Monitored SI PC104 camera card buss voltages (+3.3vdc, $+5 \mathrm{vdc},+12 \mathrm{vdc})$ stayed within specs.

- Monitored PC104 power supply current draw stayed within specs. 


\section{SI PC104 Performance Test Report}

National Ignition Facility Testing Procedure

- SI PC104 camera card visual inspection pre/post test showed

All solder joints intact

No damaged trace paths

- No crashes of SI PC104 computer or camera card were noted. Software, hardware and operating system were stable throughout test phase.

- There were no power failure or communication problems with the camera card during image acquisition cycling.

- Camera communication failures were noted on power up but were not pursued at this time.

Improper shutdown (power cycle) of SI PC104 system sometimes caused communication issues with camera card and simulator.

Recommendation is to do further testing to isolate problem. See Test Plan 2.

\subsection{Test Plan 1 Recommendations}

A "SI PC104 Usage Recommendation Memo" (Appendix E) was developed to provide guidelines to the SI PC104 User community. Spectral Instruments was informed of camera card modifications, test results and usage recommendations implemented at LLNL. Issues with the SI PC104 system were mostly attributed to SI Camera card power connector.

The recommendations included:

- Remove the LEMO power connector from the SI camera card.

- Include the Tri-M Engineering HE104+ p/s board (or comparable replacement)

- De-solder and insulate the input/output legs of the 3.3V regulator on the SI camera card

- Tap off the $+28 \mathrm{~V}$ input power to supply the fans.

-Ensure all PC-104 and PC-104+ cards conform to the PC-104 or PC104+ specification.

\subsection{Recommended additional performance tests include:}

- Run image acquisition tests with a fully modified SI PC104 Camera Card.

- Validate file protection performance of the SI PC104 Disk-on-Chip (DOC) Enhanced Write Filter (EWF) function during power down.

- Perform additional tests with power down/up and initialization of the SI PC104 to identify proper power cycling procedure 


\section{SI PC104 Performance Test Report}

\subsection{Additional Performance Stress Tests Recommended:}

- Raise ambient temperature of SI PC104 System to $40^{\circ}+20 \%$

- Vary SI PC104 System Input Power (28V) to SI specification boundaries

- Vary SI-1000 Camera Power Supply (28V) to vendor specification boundaries

- Perform a SI PC104 System Vibration test

\subsection{Test Plan 2 Results Summary:}

-Data log files associated with these tests are identified in Section 7.3 Data File Locations.

- Performance test of a fully modified camera card with continuous image acquisition cycles.

No crashes of PC104 computer

Successful performance of modified camera card during extended image acquisition cycles. No image acquisition failures due to power fail.

Successful validation of software fixes correcting Exposure/Read issue. All image acquisitions were successful using the new version of SITASJNIInterface.DLL (dated 8/9/2005). This showed no problems during Exposure/Read phase. UEC test logs showed no "CANCEL" message indicating image acquisition abort.

All SI PC104 camera card buss voltages stayed within specs

-Problem reports noted:

SI driver installation problems after power cycle. Used combination of RESTART and reboot of SI simulator to establish good state of operation.

- EWF Function Verification

Numerous power cycles (28v cutoff, Shutdown, Restart) under various conditions of PC104 System (Idle, Image Acquisition READ) did not cause file corruption.

SI PC104 System files were compared against a set of files copied to the Laptop test system prior to starting the tests. System file integrity was successfully verified after each test.

-Problem report noted:

SI driver installation problems after power cycle. Used combination of 


\section{SI PC104 Performance Test Report}

RESTART and reboot of SI simulator to establish good state of operation.

-Diagnosis of power cycle and initialization problems on SI PC104 system

SI Driver Installation was always unsuccessful after full power down sequence. Further testing showed that the SI driver will not install with the Camera Card $+3.3 v d c$ regulator removed or pins detached. Spectral Instruments states a $50 \mathrm{msec}$ minimum rise time for the $+3.3 \mathrm{vdc}$ buss. With the $+3.3 \mathrm{vdc}$ regulator detached, the PC104+ power supply $+3.3 \mathrm{vdc}$ buss rise time is inadequate $(\sim 125 \mathrm{msec})$. See Appendix $G$ for PC104 power supply waveforms showing both adequate and inadequate rise times.

With the SI camera card $+3.3 \mathrm{vdc}$ regulator fully attached, the SI driver successfully installed $100 \%$ of the time. This showed an adequate rise time $(\sim 25 \mathrm{msec})$ to meet SI specs. Spectral Instruments stated that having the $+3.3 v d c$ regulator fully attached while using the HE104+DX power supply to also provide $+3.3 \mathrm{vdc}$ will cause early component failure. This is not a long-term solution.

-PC104Working Group Recommendation

Fully attach the $+3.3 \mathrm{vdc}$ regulator onto the SI PC104 camera card.

Install and test with a PC104 format Power Supply that provides only $+5 \mathrm{vdc}$ and $+12 \mathrm{vdc}$. This allows the SI PC104 camera card to provide the +3 . 3 vdc required.

- Follow-up tests were done after implementing the PC104 Working Group Recommendations

An extended performance test (continuous image acquisition cycles) using a PC104 format power supply (Tri- M Engineering HE104-75W) providing only $+5 v d c$ and $+12 v d c$ showed successful results.

- SI driver installation was successful after every full power cycle.

During tests using the HE104+DX and SI camera card with the +3.3vdc regulator pins lifted, SI driver installation failed everytime.

During tests with the HE104-75W and SI camera card +3.3vdc regulator pins attached, SI driver installation was successful everytime ( $>5$ times in sequence). 


\section{SI PC104 Performance Test Report}

With every power cycle (1) SI driver installation was verified, camera initialization was verified, (3) and an image acquisition test was completed successfully.

- Camera card $+3.3 \mathrm{vdc} /+5 \mathrm{vdc}$ rise time measured to be adequate per $\mathrm{SI}$ requirement $(\leq 50 \mathrm{msec})$.

- Continuous cycles of image acquisition showed no image acquisition failures due to power failure or CPU performance.

- No crashes of SI PC104 computer or camera card were noted. Software, hardware and operating system were stable throughout test phase.

- There were no power failure or communication problems with the camera card during image acquisition cycling.

- All SI PC104 camera card buss voltages stayed within specs

\subsection{TEST PLAN 2 RECOMMENDATIONS}

-A "SI PC104 Usage Recommendation Update Memo" (Appendix F) was developed to provide updated guidelines based on final testing. This memo is to be sent to Spectral Instruments and the SI PC104 user community.

Do not use the Lemo power connector from the SI Gigabit I/O card. If required in tight designs, this connector can be removed. However, it is not recommended, if avoidable.

Design diagnostics using the SI PC-104+ system to include the Tri-M HE104-75W power supply board. This board supplies power to the $+5 \mathrm{vdc}$ and $+/-12 v d c$ PC-104+ busses only. The $+3.3 v d c$ buss is supplied by the SI camera card on-board +3 .3vdc regulator.

-PC104 Working Group Recommendations

Send all SI 1000 cameras and PC104 systems to SI for update to latest revision levels (hardware and software).

Update existing SI PC104 systems with latest SITASJNIInterface.dll.

Run SI PC104 performance tests with latest hardware and software available from both SI and LLNL.

Include an image acquisition performance loop test as part of LLNL system verification prior to release of newly purchased or updated $\mathrm{SI}$ PC104 systems.

\subsection{Summary}

This draft provided by the editor is intended for review by the author and reviewers listed on the signature page. Please mark any additions or omissions or other edits with red pen and remit your marked-up copy to the author. Contact the editor for the most up-to-date version if you need a Word file. 


\section{SI PC104 Performance Test Report}

- Test Plan 1 results showed successful CPU and camera card performance with the PC104+ power supply. Occasional failures were noted with image acquisition and power up initialization of the SI PC104 with the SI camera. The image acquisition issue was well understood and fixed via software. The power up initialization required further testing implemented in Test Plan 2. A SI PC104 Usage Recommendation memo was sent out to the SI PC104 user community.

- Final Test Plan 2 results showed successful performance of a modified camera card (only LEMO power connector removed) during continuous image acquisition cycles. The updated SITASJNIInterface.dll (dated 8/9/2005) provided successful image acquisition during continuous Exposure/Read cycles with no "CANCEL" messages noted. The EWF function was verified to maintain system file integrity after multiple and various methods of power down. Camera power up initialization issues (SI driver installation) were solved by installing a PC104 format power supply module supplying only $+5 \mathrm{vdc}$ and $+12 \mathrm{vdc}$ allowing the $\mathrm{SI}$ camera card on-board regulator to supply the $+3.3 \mathrm{vdc}$ buss.

- Test results were followed up with SI PC104 Usage Recommendation memos to the SI PC104 user community. Two recommendation memos are attached. Memo \#1, Appendix E "SI PC104 Usage Recommendation Memo", developed after Test Plan 1 and Memo \#2, Appendix F "SI PC104 Usage Recommendation Memo Update", developed after Test Plan 2.

- Overall test results showed successful implementation of solutions to SI PC104 issues encountered during initial tests and operations. The SI PC104 system is now a reliable, robust system capable of extended operations in the NIF facility.

- Additional performance stress tests are recommended to more fully address possible operational issues in NIF. These include raising the ambient temperature of SI PC104 System to $40^{\circ}+20 \%$, varying the SI PC104 System Input Power (28V) to SI specification boundaries, varying the SI-1000 Camera Power Supply (28V) to vendor specification boundaries, and performing a SI PC104 System vibration test.

\subsection{Test Participants}

List, by position, the personnel required to perform the test.

\begin{tabular}{|l|l|}
\hline Name & Position \\
\hline Sam Montelongo & PC104 Working Group Lead \\
\hline Jarom Nelson & PC104 Software Developer \\
\hline James Moody & SI Camera Expert \\
\hline Ken Piston & Electronics Support \\
\hline Joe Holder & Test Plan Reviewer \\
\hline
\end{tabular}

This draft provided by the editor is intended for review by the author and reviewers listed on the signature page. Please mark any additions or omissions or other edits with red pen and remit your marked-up copy to the author. Contact the editor for the most up-to-date version if you need a Word file. 


\title{
SI PC104 Performance Test Report
}

\subsection{Location of Tests}

\author{
List, Location of tests
}

\begin{tabular}{|l|l|}
\hline Building/Room & Test carried out at this location \\
\hline Building 391, Room B350 & Test Plan 1 \\
\hline Building 391, Room B350 & Test Plan 2 \\
\hline & \\
\hline
\end{tabular}

\subsection{Test Data Locations}

The following table should list data files (e.g., spreadsheets, tables, images) associated with the executed CTP. Ideally, such data files are uploaded to the corresponding CTR record in ECMS. In any event, list each file's location (e.g., "ECMS," or "John Smith's hard drive") in the table below. Include a brief description of each file's content.

\begin{tabular}{|c|c|c|}
\hline File Name or Label & File Location & Brief Description of Data in the File \\
\hline 33RegConfigComparison.xls & \begin{tabular}{|l|} 
TDServerlPC104TestDataL \\
ogFiles\BussWaveforms
\end{tabular} & $\begin{array}{l}\text { Data and chart showing comparison of }+3.3 \mathrm{vdc} \\
\text { buss signal at startup measured with SI camera } \\
\text { card regulator pins attached and detached. }\end{array}$ \\
\hline HE104+DX_Only5and3.xls & \begin{tabular}{|l|} 
TDServerlPC104TestDataL \\
ogFiles\BussWaveforms
\end{tabular} & $\begin{array}{l}\text { Data and chart showing }+3.3 v d c \text { and }+5 v d c \text { signals } \\
\text { measure at power up coming directly out of the } \\
\text { HE104+DX power supply. }\end{array}$ \\
\hline $\begin{array}{l}\text { HE104- } \\
\text { 75W_System_3\&5_Chart.xls }\end{array}$ & $\begin{array}{l}\text { TDServerlPC104TestDataL } \\
\text { ogFiles\BussWaveforms }\end{array}$ & $\begin{array}{l}\text { Data and chart showing }+3.3 \mathrm{vdc} \text { and }+5 \mathrm{vdc} \text { signals } \\
\text { measure at power up coming directly out of the } \\
\text { HE104-75W power supply. }\end{array}$ \\
\hline $\begin{array}{l}\text { SITASJNIINterface062204_H } \\
\text { E104+DX_UecTestLog-Wed- } \\
\text { Jul-13-13-15-33-587.txt }\end{array}$ & $\begin{array}{l}\text { TDServerlPC104TestDataL } \\
\text { ogFilesICameraCardPerfor } \\
\text { mance }\end{array}$ & $\begin{array}{l}\text { UEC test log with "ACQ CANCELED" messages } \\
\text { indicating image acquisition failure while using } \\
\text { SITASJNIInterfac.dll dated 6/22/04. }\end{array}$ \\
\hline $\begin{array}{l}\text { HE104+DX_UecTestLog-Thu- } \\
\text { Sep-15-10-38-55-280.txt }\end{array}$ & $\begin{array}{l}\text { TDServerlPC104TestDataL } \\
\text { ogFilesiCameraCardPerfor } \\
\text { mance }\end{array}$ & $\begin{array}{l}\text { UEC test log showing no "ACQ CANCELED" } \\
\text { messages while using SITASJNIInterfac.dll dated } \\
\text { 8/9/05 and HE104+DX power supply. }\end{array}$ \\
\hline $\begin{array}{l}\text { BussV_HE104+DX_LogFile_0 } \\
\text { 9-15-05_10-57-55.txt }\end{array}$ & $\begin{array}{l}\text { TDServerIPC104TestDataL } \\
\text { ogFilesICameraCardPerfor } \\
\text { mance }\end{array}$ & \begin{tabular}{|l} 
IO log file monitoring PC104 buss voltages and \\
temperature while using HE104+DX power supply.
\end{tabular} \\
\hline $\begin{array}{l}\text { HE104-75W_UecTestLog-Thu- } \\
\text { Nov-10-09-28-00-177.txt }\end{array}$ & $\begin{array}{l}\text { TDServerlPC104TestDataL } \\
\text { ogFilesICameraCardPerfor } \\
\text { mance }\end{array}$ & \begin{tabular}{|l|} 
UEC test log showing no "ACQ CANCELED" \\
messages while using SITASJNIInterfac.dll dated \\
$8 / 9 / 05$ and HE104-75W power supply. \\
\end{tabular} \\
\hline $\begin{array}{l}\text { BussV_HE104- } \\
\text { 75W_LogFile_11-10-05_09-46- } \\
\text { 09.txt }\end{array}$ & $\begin{array}{l}\text { TDServerlPC104TestDataL } \\
\text { ogFilesICameraCardPerfor } \\
\text { mance }\end{array}$ & $\begin{array}{l}\text { IO log file monitoring PC104 buss voltages and } \\
\text { temperature while using HE104-75W power supply }\end{array}$ \\
\hline $\begin{array}{l}\text { SITASJNIInterface080905_H } \\
\text { E104+DX_UecTestLog-Mon- } \\
\text { Nov-07-09-58-47-092.txt }\end{array}$ & $\begin{array}{l}\text { TDServerlPC104TestDataL } \\
\text { ogFilesICameraCardPerfor } \\
\text { mancelExposureReadTest }\end{array}$ & $\begin{array}{l}\text { UEC test log showing no "ACQ CANCELED" } \\
\text { messages while using SITASJNIInterfac.dll dated } \\
\text { 8/9/05 and HE104+DX power supply. }\end{array}$ \\
\hline
\end{tabular}




\title{
SI PC104 Performance Test Report
}

\author{
National Ignition Facility Testing Procedure
}

NIF- \# 0112966-AA

\begin{tabular}{|c|c|c|}
\hline $\begin{array}{l}\text { SITASJNIInterface062204_H } \\
\text { E104-75W_UecTestLog-Thu- } \\
\text { Nov-10-09-28-00-177.txt }\end{array}$ & $\begin{array}{l}\text { TDServerlPC104TestDataL } \\
\text { ogFilesiCameraCardPerfor } \\
\text { mancelExposureReadTest }\end{array}$ & $\begin{array}{l}\text { UEC test log showing no "ACQ CANCELED" } \\
\text { messages while using SITASJNIInterfac.dll dated } \\
6 / 22 / 04 \text { and HE104-75W power supply. }\end{array}$ \\
\hline $\begin{array}{l}\text { SITASJNIInterface080905_H } \\
\text { E104-75W_UecTestLog-Fri- } \\
\text { Nov-11-10-29-06-502.txt } \\
\end{array}$ & $\begin{array}{l}\text { TDServerlPC104TestDataL } \\
\text { ogFilesICameraCardPerfor } \\
\text { mancelExposureReadTest }\end{array}$ & $\begin{array}{l}\text { UEC test log showing no "ACQ CANCELED" } \\
\text { messages while using SITASJNIInterfac.dll dated } \\
\text { 8/9/05 and HE104-75W power supply. }\end{array}$ \\
\hline $\begin{array}{l}\text { PC104 DOC System Files } \\
\text { Folder }\end{array}$ & $\begin{array}{l}\text { TDServerIPC104TestDataL } \\
\text { ogFilesIEWFPerformancelfi } \\
\text { les to compare }\end{array}$ & $\begin{array}{l}\text { This folder contains files copied from the PC104 } \\
\text { Disk-On-Chip prior to beginning EWF tests. }\end{array}$ \\
\hline file_compare_repeat.cmd & $\begin{array}{l}\text { TDServerIPC104TestDataL } \\
\text { ogFilesIEWFPerformance }\end{array}$ & $\begin{array}{l}\text { DOS command file comparing PC104 DOC system } \\
\text { files with the "files_to_compare" folder and creating } \\
\text { a log file with comparison result. }\end{array}$ \\
\hline \begin{tabular}{|l} 
fc_output_092305.txt \\
fc_output_092605.txt \\
fc_output_092605a.txt \\
fc_output_092805.txt \\
fc_output_092905.txt \\
fc_output_093005.txt \\
\end{tabular} & $\begin{array}{l}\text { TDServerIPC104TestDataL } \\
\text { ogFilesIEWFPerformance }\end{array}$ & $\begin{array}{l}\text { Comparison results from issuing } \\
\text { "file_compare_repeat.cmd" after power cycling the } \\
\text { PC104 system using various methods. }\end{array}$ \\
\hline $\begin{array}{l}\text { PC104_HE104- } \\
\text { 75W_PowerCycle_UecTestLo } \\
\text { g-Thu-Nov-10-09-28-00- } \\
\text { 177.txt }\end{array}$ & $\begin{array}{l}\text { TDServerlPC104TestDataL } \\
\text { ogFilesIPowerCyclePerform } \\
\text { ance }\end{array}$ & $\begin{array}{l}\text { UEC test log showing no "ACQ CANCELED" } \\
\text { messages while using HE104-75W power supply. } \\
\text { This file also logged messages captured after } \\
\text { power cycle tests showing no problems occurring } \\
\text { with camera initialization or image acquisition. }\end{array}$ \\
\hline
\end{tabular}

\subsection{Terminology}

List all abbreviations, initialisms, and acronyms used in the test procedure. Define special or nonstandard terms used where understanding of such terms is important to successful execution of the test.

\begin{tabular}{|l|l|}
\hline Term & Definition or Full Spelling \\
\hline SI & Spectral Instruments \\
\hline PC104 & Industry standard for PC104 systems \\
\hline DC & Diagnostic Controller \\
\hline EWF & Enhanced Write Filter \\
\hline UEC & Universal Embedded Code \\
\hline & \\
\hline
\end{tabular}

\subsection{Listing of Applicable Reference Documents}

List operations or other procedures and documents that were used to perform the test procedures or analyze data 


\begin{tabular}{|c|c|c|}
\hline \multicolumn{2}{|c|}{ SI PC104 Performance Test Report } & 1) \\
\hline National Ignition Facility Testing Procedure & NIF-\# 0112966-AA & $\begin{array}{c}\text { Page } 13 \text { of } \\
53\end{array}$ \\
\hline
\end{tabular}

during the testing procedure (for example, installation or interconnect drawings and/or standard operating procedures).

\begin{tabular}{|l|l|}
\hline Document \# & Title \\
\hline Appendix A & Test Plan 1 for the SI-1000 PC104 Camera Card \\
\hline Appendix C & Test Plan 2 for the SI-1000 PC104 Camera Card \\
\hline Appendix E & SI PC104 Usage Recommendation Memo \\
\hline & \\
\hline
\end{tabular}

\subsection{Appendix}

The following appendices include test plans, test plan results, recommendation memos, and various PC104 buss waveforms.

Test Plan 1, Test Plan 2, Test Plan 1 Results, and Test Plan 2 Results were presented to the PC104 Working Group team as indicated.

The SI PC104 Recommendation Usage Memo was sent out to the SI PC104 User Community. The SI PC104 Recommendation Update Memo will be sent out to the user community when complete.

PC104 buss waveforms show the various waveforms captured to help isolate the SI driver installation problem. 


\section{SI PC104 Performance Test Report}

\subsection{Appendix A: Test Plan 1 for the SI-1000 PC104 Camera Card}

- Test Plan 1 was presented to the PC104 Working Group Team on June 10, 2005 prior to the beginning of testing. The presentation included

- Test Overview

Test Goals

Test Approach

Test System

Hardware Components

Software Components

UEC Test Program

Diamond I/O Vendor Software

Test Area Configuration

Stress Test Plan

Stress Test Expectations

Secondary Tests

Personnel

Test Schedule 


\section{SI PC104 Performance Test Report}

SI PC104 Working Group June 10, 2005

Stress Test Plan for the SI-1000 PC104 Camera Card

\section{Test Plan Review Topics}

- Test Overview

> Test Goals

$>$ Test Approach

> Test System

- Hardware Components

- Software Components

- LEC Test Program

- Diamond $1 / 0$ Vendor Software

- Test Area Configuration

> Stress Test Plan

$>$ Stress Test Expectations

> Secondary Tests

- Personnel

- Test Schedule

- Test encompasses:

- Monitor and verify SI PC104 system operation (in particular the SI camera card) over an extended period of time using a PC104 power supply module to provide power.

- Monitor/record SI PC104 camera card busses (voltages) and current draw.

- Monitor/record SI PC104 camera card temperature at selected areas.

- Monitor/record PC104 power supply card temperature at selected areas.

- Monitor/document SI PC104 system failures.

- Use Test Program to continuously cycle the SI PC104 system thru image acquisition cycles.
- Implement a continuous cycle image acquisition stress test on a SI PC104 Camera card configured to receive its power from a PC104 power supply module.

- Identify and fix failure modes of the SI PC104 Camera Card when stressed over an extended period of time.

- Provide guidelines for using the SI PC104 Camera card in diagnostic configurations

- Develop CM process for testing new PC104 system components ( $\mathrm{HW} / \mathrm{SW}$ ) prior to release for use.

Jane 10,2005 
SI PC104 Performance Test Report

\section{Test Approach}

- Use current NIF tested

- UEC code for SI PC104 System

- DSP file for S1-1000

- Windows driver for $S I-1000$

- Use UEC Test program to drive system through con tinuous non-triggered image acquisiti on cycles

(using loop and delay functions) for a period of 5 days.

- Test PC, SI PC-104 Embedded Controller and PC104

Test Stand will communicate over network link

- Use Diamond $1 / 0$ card and vendor software to

monitor/record buss voltage and temp parameters

- PC104 Power Supply (TEMP)

- S1 PC104 Camera Card (Buss Voltages, TEMP, Current)

- Use DANTE $\mathrm{CCl}$ as basis for test system

- System available

- Extreme config known to have had problem with SI PC104 system

hune 10,2005
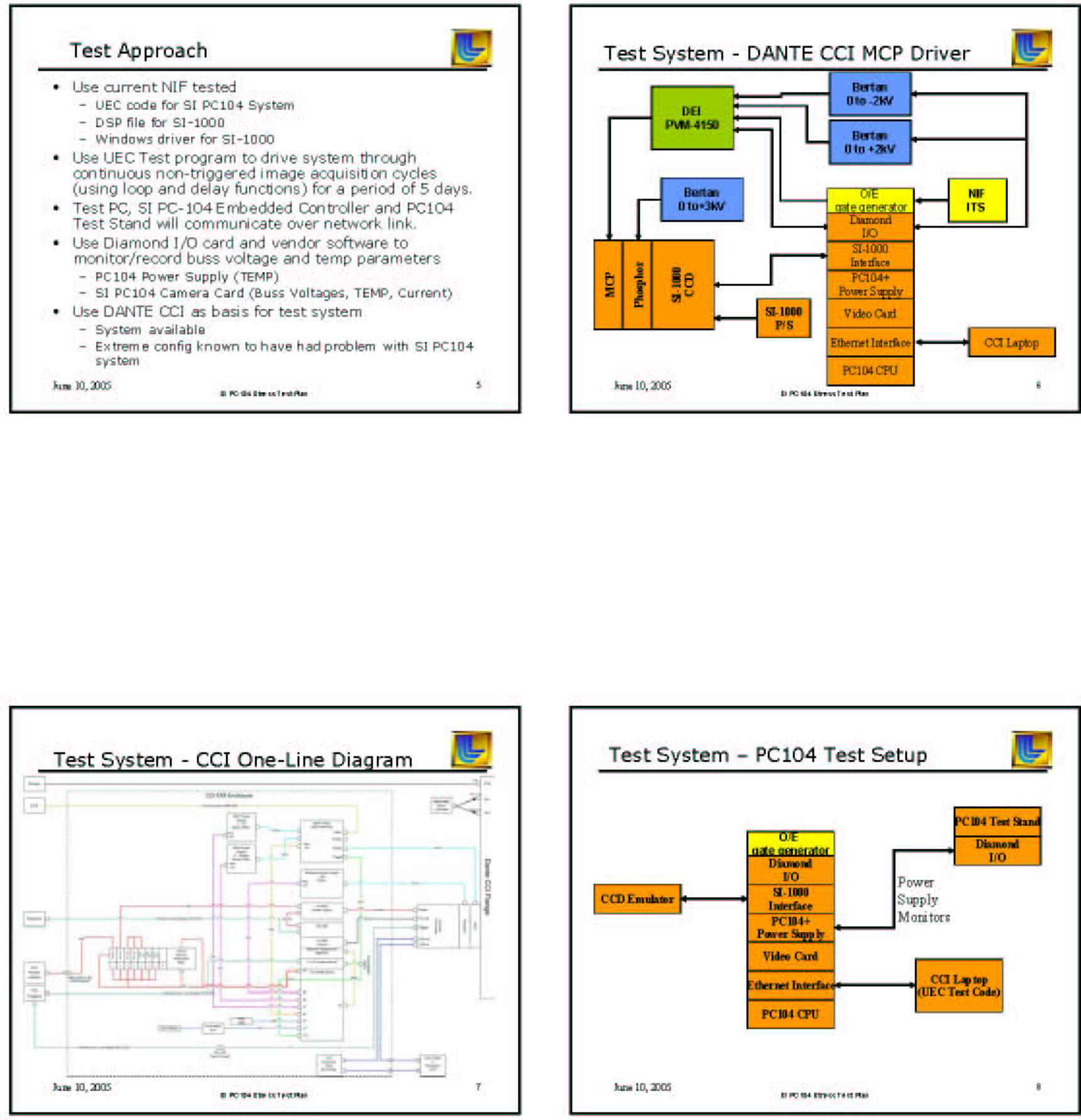

Test System - PC104 Test Setup

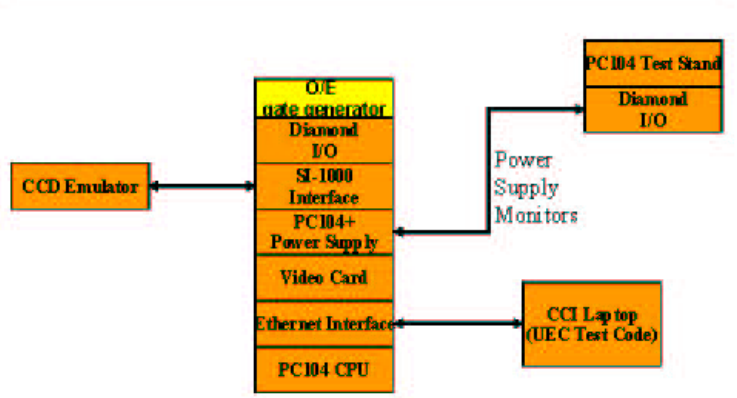

flane 10,2005 
SI PC104 Performance Test Report

\section{Test System - Diamond Systems I/O Card (L)}

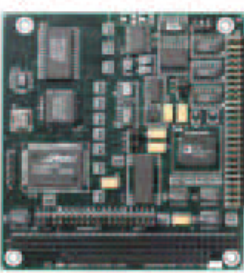

og Input

+16 single-ended i 8 differential inputs

+16 -bit AuD resolution

+ Frogrammable input ranges with maximum range of $+M$ DV Analog Output

+4 optional analog outputs

+ Fixed and user-programmable output ranges

+ Simultaneous update

Digital vo

+ 8 dedicated dightal outputs TL compatible

+8 dedicated digital inputs, TTL compatible

CounterTimers

+ 132 -bit countertimer for AND sampiling rate control

+1 16-bit countertimer for user counting and timing functions

hine 10,2005

+ Programmable clock source for user countertimer

an pores strotortan
Hardware Components

- SI PC104 Camera Card

- Lift/insulate pins on $3 v$ regulator

- Do not remove power connector/components

- Temp Sensors (Qty TBD) at selected locations

- Buss Voltage monitor (3.3v, 5v, 12v)

- Current draw monitor (input only)

- PC104 Power Supply

- Include PC104 P/5 module in stack (DONE)

- Route $28 \mathrm{v}$ power to PC104 P/S (DONE)

- TEMP Sensor (1)

- SI-1000

- s1-1000 Em ulator with Optical in terface cable

- Power load not required, power not supplied via SI PC104

- Triggers not required

- PC104 Test Stand

- D iam ond $y / O$ board interfaced to monitor param eters

hane 10,2000

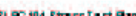

\section{Software Components}

- Test Station PC

- DANTE CCI Laptop

- UEC Test Program

- 1 week development (loop/delay function)

- Configuration setup

- PC104 Test Stand

- Diamond $[/ / O$ vendor code - 1 week develop/configure

- SI PC104 Embedded Controller

- UEC version 1.1.6 (on-hand)

- DSP version D (on-hand)

- No PC104 firmware updates required
UEC Test Program - J. Nelson

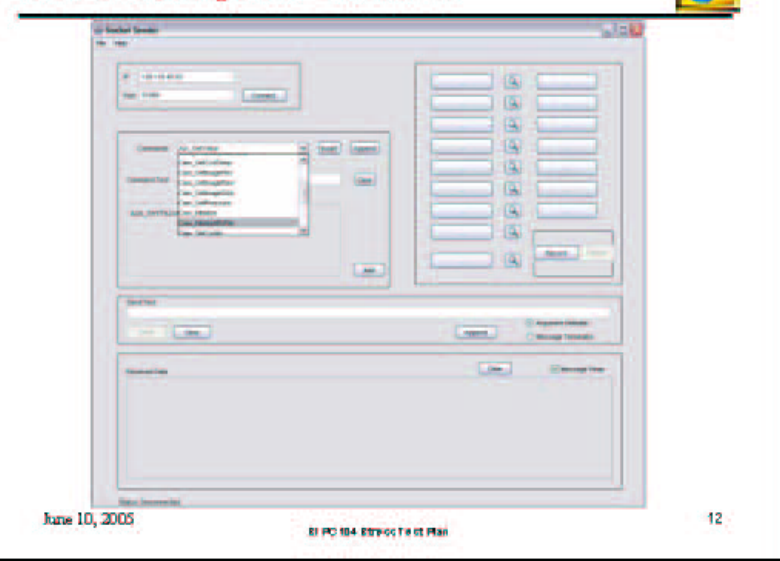




\section{SI PC104 Performance Test Report}

\section{Stress Test Plan}

- Run continuous cycles of image acquisition

- Use same configuration during all testing

- Aoquired image files are not saved

- Continuous test over a period of 5 days mini mum

- Monitor/record SI PC104 camera card buss voltages

- Monitor/record SI PC104 camera card temperature at selected locations

- Monitor/record PC104 power supply current draw

- Record failures

- Inspect PC104 camera card pre/post test - Solder joints

- Trace paths

- Evaluate results and write guidelines

fune 10,2005

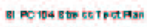

Test Area Configuration

- B391 Rm B350

- DWTL: K.Piston

- IWS: T.Thomas

- Dante CCI System

- J.Holder/K.Piston

- PC Test Station

- K.Piston

- PC104 Test Stand

- J. Nelson

- Network Communication

- K.Piston, J. Nel son

\section{Stress Test Expectations}

- Run continuous cycles of image acquisition - No image acquisition failures

- Monitor/record SI PC104 camera card buss voltages - Buss voltages stay within specs

- Monitor/record SI PC104 camera card temperature at selected locations

- Temperature stays within specs

- Monitor/record PC104 power supply current draw

- Current draw stays within specs

- Record failures

- No unexplainable failures

- Inspect PC104 camera card pre/post test

- Solder joints: All solder joints solid

- Trace paths: No damaged trace paths

Thene 10,2005

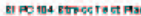




\section{SI PC104 Performance Test Report}

\section{Secondary Tests}

- Power cycling SI PC104 system

- Cable 'rattling', vibration, shock, temperature changes

- Install and test SI PC104 with KONTRON motherboard

- Test with modified SI PC104 Camera Card

- Remove power connector/components

- Monitor DMA buss signals with analyzer over extended stress test

Equipment Resources

- PC Designated as Test Computer

- DANTE Diagnostic Instrumentation

- PC104 Test Stand

- SI-1000 Emulator and Optical Interface Cable
Test Schedule

- B391 Test System Configuration and Setup

- June 13-17 K.Piston, J.Nelson

- UEC Test Program develop/setup: JNelson

- S1 PC104 Camera Card Mods/Prep: J.Moody

- S1 PC104 Power Supply Prep: K.Piston

- Diam ond I/O setup/SW config: K.Piston

- Stress Test testing

- June 20-30 J.Nelson, K. Piston

- Evaluate Test Results

- July 1-8 J.Moody, S.Montelongo, J.Nelson, K. Piston

- Write SI PC104 Camera Card Usage Guideline

- July 11-22 S.Montelongo

- Develop SI PC104 Component (HW/SW) CM Process

- July 11-22 J.Moody

flane 10,2005

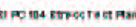




\section{SI PC104 Performance Test Report}

\subsection{Appendix B: Test Plan 1 (Results for the SI-1000 PC104 Camera Card)}

Test Plan 1 Results were presented to the PC104 Working Group Team on August 11, 2005. The presentation included

Test Overview

Test Goals

Test Implementation

Modified Hardware

Test System Configuration

Test Plan

Test Results

UEC Test Program Implementation and Results

I/O Test Implementation and Results

SI-PC104 Camera Card Inspection

SI PC104 Usage Recommendation

Additional Performance Tests

Recommended Stress Tests

Personnel Involved

Test Schedule 


\section{SI PC104 Performance Test Report}
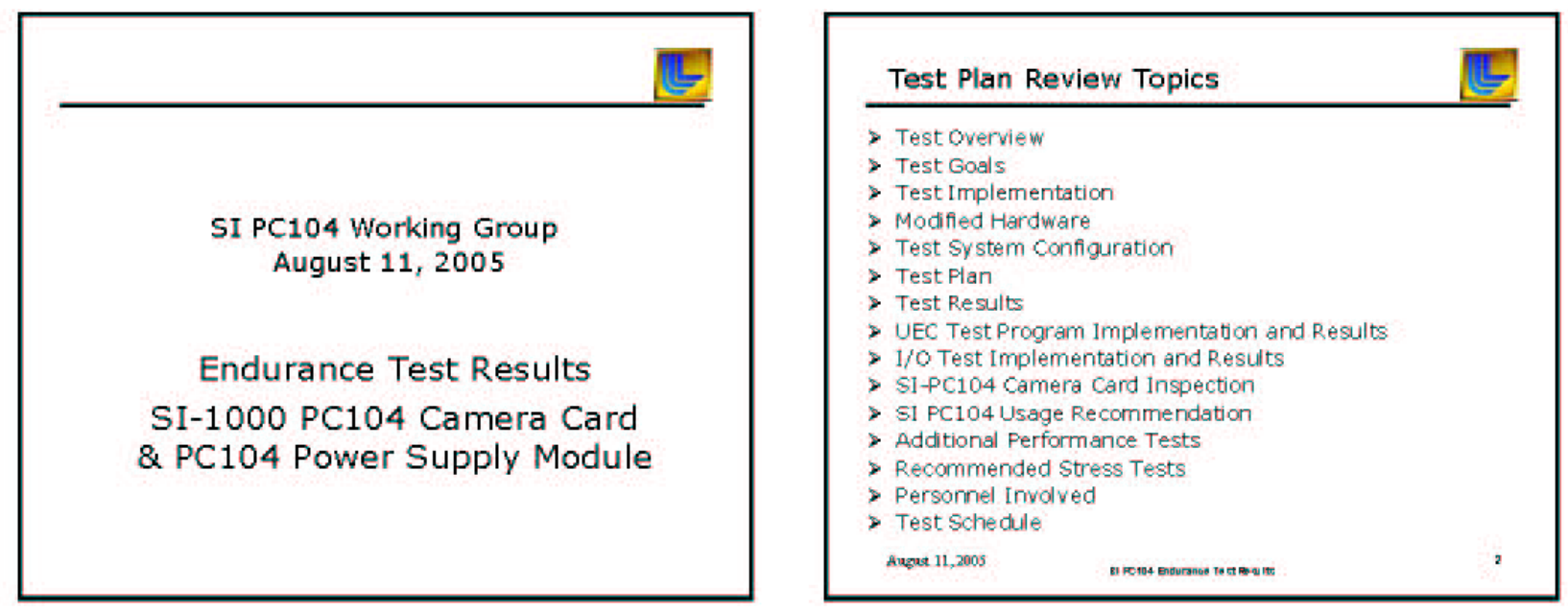

Test Overview

- Monitor and verify SI PC104 system operation (in particular the SI camera card) over an extended period of time using a PC104 power supply module to provide power.

- Monitor/record SI PC104 camera card buss vol tages and current draw.

- Monitor/record PC104 power supply module temperature.

- Monitor/document SI PC104 failures.

- Use Test Program to continuously cycle the $\mathrm{SI}$ PC104 system thru image acquisition cydes.

Angert 11,2005

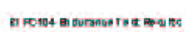
$s$
Test Goals

- Implement an endurance test of a S1 PC104 Camera card configured to receive its power from a PC104 power supply module.

- Identify and fix failure modes of the S1 PC104 Camera Card when used over an extended period of time.

- Provide recommendation for using the SI PC104 Camera card in diagnostic configurations

- Develop CM process for testing new PC104 system components ( $\mathrm{HW} / \mathrm{SW}$ ) prior to release for use.

Angert 11,2005

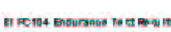


SI PC104 Performance Test Report

\section{Test Implementation}

- Test Dates: $6 / 27 / 05-7 / 6 / 05,8 / 1 / 05-8 / 5 / 05$

- Test Location:B391 Rm B350

- Test System: DANTE CCI with SI-1000 Simulator, PC104

Test Stand, modified 51 Camera card, PC104 P/S

- UEC code for SI PC104 System, Ver. 1.1.6

- DSP file for S1-1000, Reo.D

- Used UEC Test program to drive system through continuous non-triggered image acquisition cycles.

- Used Diamond I/O card to monitor/record buss voltage and temp parameters

- PC104 Power Supply (TEMP)

- SI PC104 Camera Card (Buss valtages, Current)

- Used DANTE CCl as basis for test systern

- Configuration known to have had problem with SI PC104 system

Augat 11,2005
Test System - PC104 Test Setup

Anguat 11,2005

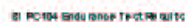
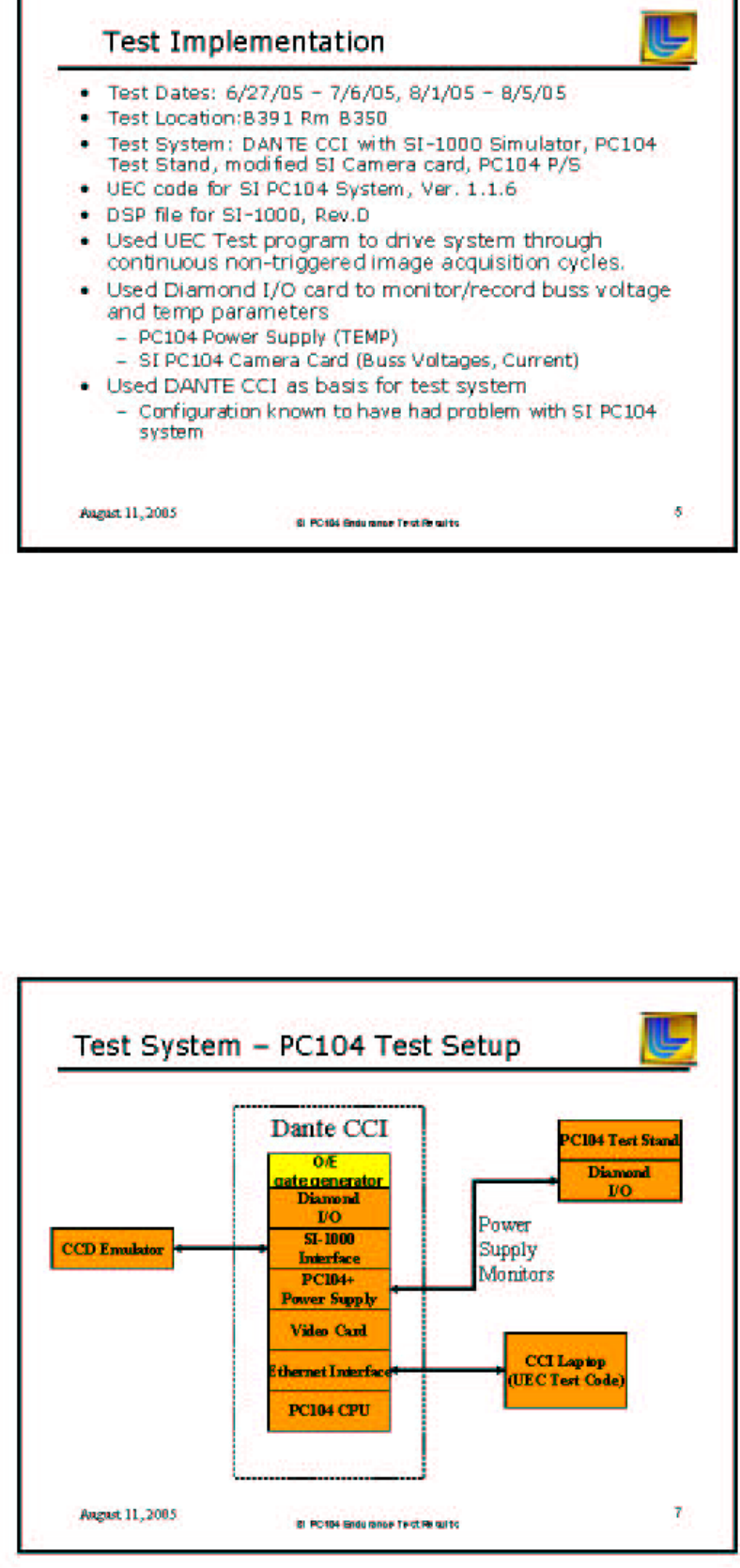

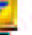$$
\text { sit }
$$

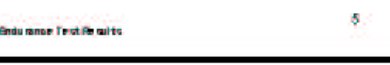

Modified Hardware Components

- SI PC104 Camera Card

- Lfted pins on $3 v$ regulator

- Did not remove power connector/components

- Buss voltage monitors (3.3v, 5v, 12v)

- Current draw monitor (input only)

- PC104 Power Supply

- TEMP sensor (1 ea)

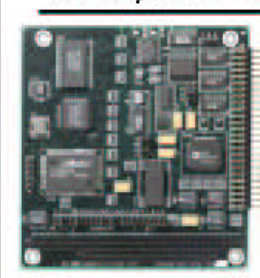

Aralog input

+16 single-ended $/ 8$ differential inouts

+16-bit AO resol ution

+ Program ma ble input ranges whth maximum range of $+40 \mathrm{~V}$

Analog Oưut

+4 optional analog outputs

+ Fixed and user-progran mable output ranges

Digital 10

+8 dedicated digital outputs, TTL compatible

48 dedicated digital inputs, TTL compatible

CounteriTimers

+1 32-bit courtertimer for A.D sampl ing rate control

+116 -bit courtertimer for user counting and timing functions

+ Progranmable dock source for user countertimer
+ Sim ultaneous update 
SI PC104 Performance Test Report

\section{TRI-M HE104+DX Power Supply}

\section{HE104-DX}

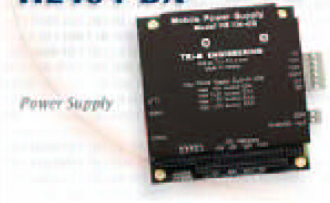

August 11,2005

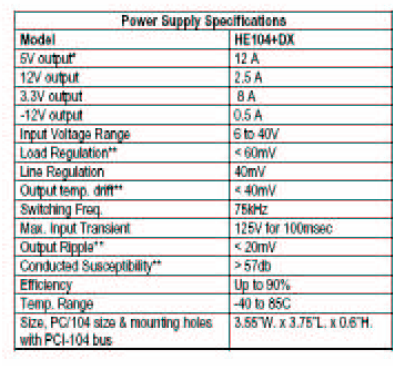
SEE, PCH104 size
with PCL1 104 bus

\section{Endurance Test Plan}

Run continuous cycles of image acquisition

- Use same configuration during all testing

- Acquired image files not saved

- Test over a period of $\sim 10$ days

- Monitor/record SI PC104 camera card buss voltages

- Monitor/record PC104 power supply current draw

- Record failures

- Inspect PC104 camera card post test

- Solder joints

- Trace paths

- Evaluate results and write recommendation

\section{Endurance Test Results}

- Run continuous cycles of image acquisition

- No image acquisition failures due to power failure

- Monitor/record SI PC104 camera card buss voltages

- Buss voltages stayed within specs

- Monitor/record PC104 power supply current draw

- Current draw stayed within specs

- Record failures

- No unexplainable failures

- Inspect PC104 camera card pre/post test

- Solder joints: All solder joints solid

- Trace paths: No damaged trace paths
UEC Test Program Implementation

- Test Loop

- Enable Digital and Analog outputs, wa it $60 \mathrm{Sec}$

- Acquire image (takes about $20 \mathrm{sec}$.), wait $50 \mathrm{Sec}$

- Abort image (in case of failure), wait $10 \mathrm{Sec}$

- Wait $60 \mathrm{Sec}$

- Disable Analog outputs, wait $60 \mathrm{Sec}$

- Disable Digital outputs, wait $60 \mathrm{Sec}$

- One image every 5 minutes
Auguat 11, 2005

SI PC 104 Endronos Tert Resuts
August 11,2005

SI PC104 Endurance Test Result: 
SI PC104 Performance Test Report

\section{UEC Test Program Results}

- No crashes of computer or camera card

- Software, hardware and operating system were stable throughout test phase.

- No power failure or communication problems with camera card.

- I mage acquisition failure noted

- Did not happen consistenty (average about 1 in 10)

- Due to Exposure Time query issue

- If exposure time queried during readout, camera may

abort image acquisition. Confirmed by vendor.

- LLNLL UEC software to be fixed.

- Communication failure on startup

- Improper shutdown (power cyde) of SI PC104

system some times caused communication issues

with camera card and simulator.

- Can be avoided by using proper restartishutdown.

Angert 11,2009

Further testing required to isolate problem

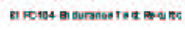

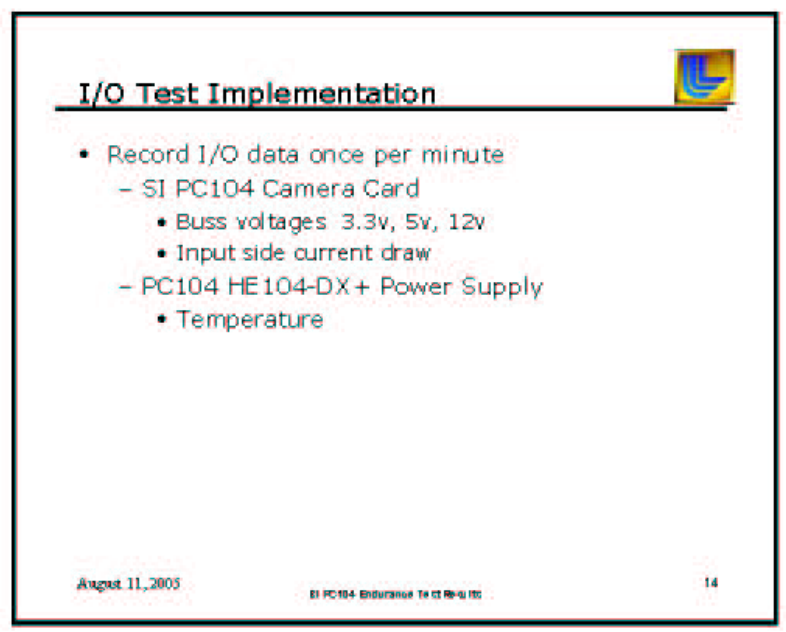

Power Supply Output $+5 v d c$

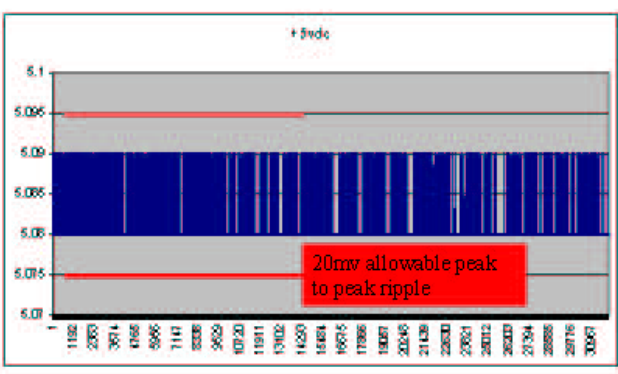

Angere 11,2005

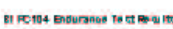




\section{SI PC104 Performance Test Report}

Power Supply Output +12 vdc

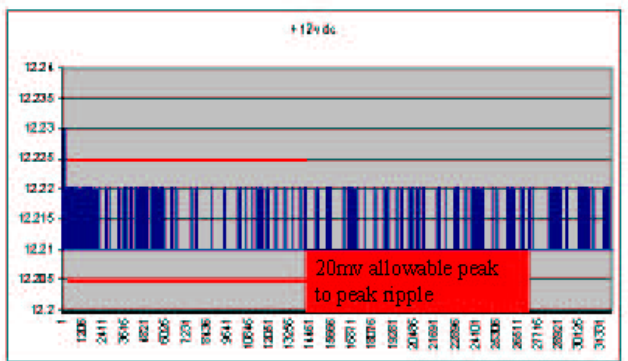

Anegr 11,2005

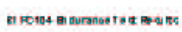

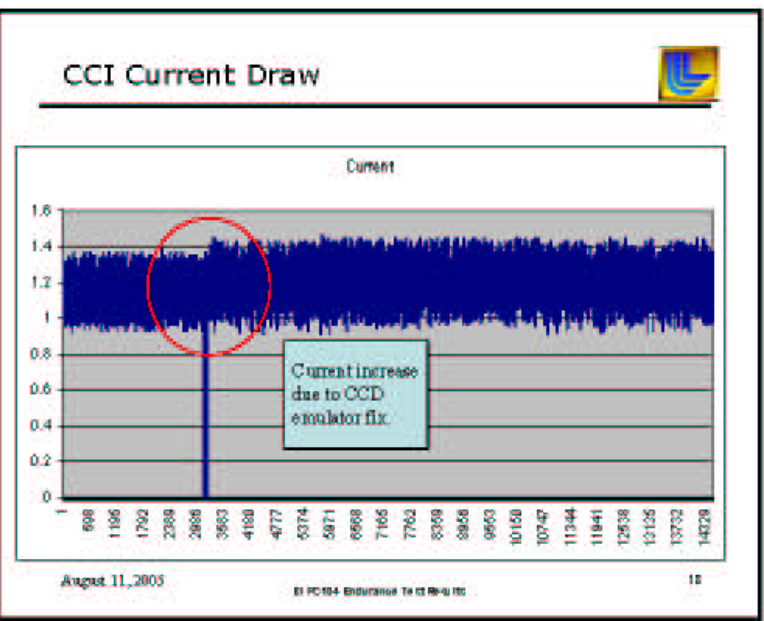

\section{CCI +25 vdc Input From Agilent}

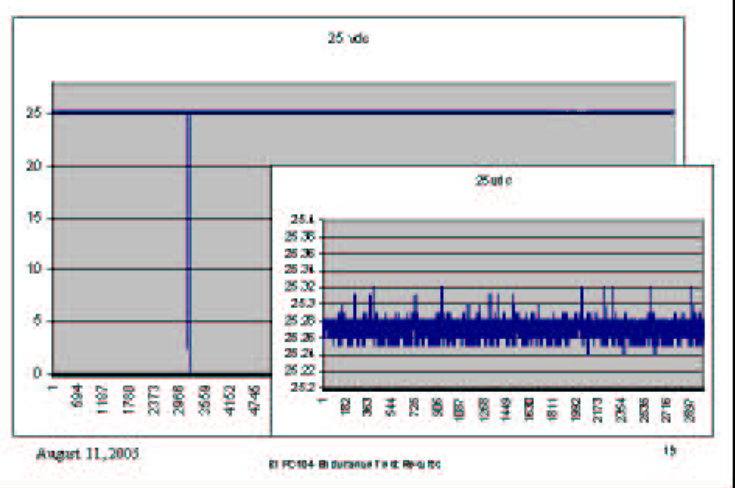

\section{HE104+DX temperature}

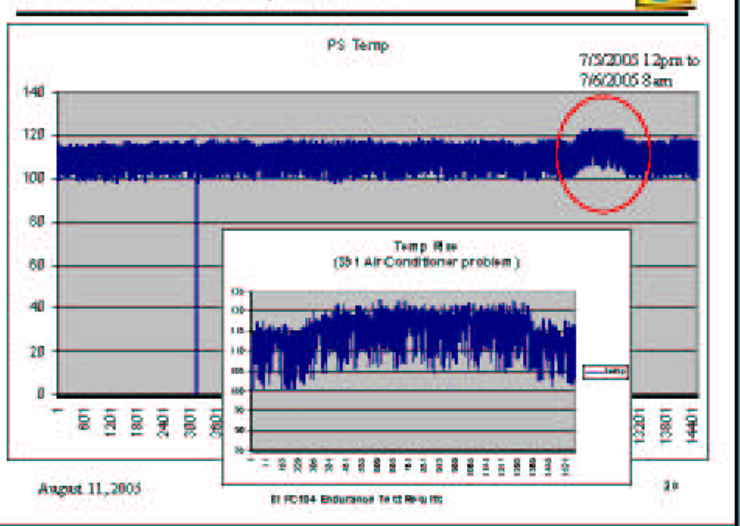


SI PC104 Performance Test Report

PC104 I/O Test System - Results
- SI PC104 Camera Card buss
voltages and current draw
performed within specifications.
- HE104+DX power supply
performed within specifications.
- Throughout testing the PC104
under test was power cycled
several times to fix issues with
the CCD emulator.
During testing the building air
conditioning system failed
raising the room temperature
slightly with no noticeable
effects on the PC104.
with a 1 second log sample. Data
shed no differences from previous
tests at 1 minute sample rate.

SI PC104 Usage Recommendation

- Issues with the SI PC104 system mostly attributed to SI Camera card power connector.

- Recommendation

- Remove the Lemo power connector from the SI camera card.

- Include the Tri-M Engineering HE104+ p/s board.

- De-solder and insulate the input/output legs of the

$3.3 \mathrm{~V}$ regulator on the SI camera card

- Tap off the $+28 \mathrm{~V}$ input power to supply the fans.

- Ensure all PC-104 and PC-104+ cards conform to the
PC-104 or PC104+ specification.

Spectral Instruments informed of camera card modifications, test results and Usage Recommendation implemented at LLNL. 


\section{SI PC104 Performance Test Report}

Recommended Stress Tests

- Raise ambient temperature of SI PC104 System top $40^{\circ}+20 \%$

- Vary SI PC104 System Input Power (28V) to specs

- Vary SI-1000 Camera Power Supply (28V) to specs

- SI PC104 System Vibration

Verify image quality pre/post each test.

Angers 11,2005

Test Schedule

- Test System Configuration and Setup : June 13-27

- UEC Test Prouram develop/setupi 1. Nel son

SI PC104 Camera Card Mods/Prep: J.Moody

- SI PC104 Power Supply Prep: K.Piston

- Diamand 1/O setup/SW bonfig: K. Piston

- Stress Test 1mpiementation : June 27-July 6

- 1. Nelson, K. Piston

- Evaluate Test Results : July 7-14

- 1.Nelson, K.Piston, 1.Moordy, S.Montelongo

- Present-Test Results : July 15

- SI PC104 Usage Recommendation Memo

- Send out in August J,Moody, S.Montelongo

- Develop SI PC104 CM Test Process (HW/SW)

- August 2005 J.Nelson, S.Montelongo

- URL Report capturing tests, results and recommendations

- September 20055 Mantelongo

Angerst 11,2005

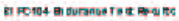

Personnel Involved with Test

- Jarom Nelson, Software support

- Ken Piston, Electronics support

- Jim Moody, SI-1000 support

- Sam Montelongo, Test Plan Manager 


\section{SI PC104 Performance Test Report}

\subsection{Appendix C: Test Plan 2 (The SI-1000 PC104 Camera Card)}

Test Plan 2 was presented to the PC104 Working Group Team on September 13, 2005 prior to the beginning of testing. The presentation included

Test Overview and Goals

Test Approach and System Configuration

Hardware and Software Components

Performance Test Plan

Resources and Test Schedule

The test to "Validate performance of a PC104 KONTRON motherboard CPU" was deemed as NOT APPLICABLE for this type of testing. The KONTRON motherboard does not support a Disk-On-Chip architecture as does the Tillamook PPM-TX PC104 CPU module delivered with the SI PC104. This would have called for a design change to the SI PC104 configuration and would require additional time to test. The KONTRON motherboard shall be tested at a later date as part of the Instrument Based Controller design. 


\section{SI PC104 Performance Test Report}

\begin{tabular}{|c|}
\hline Test Plan (2) for the \\
SI-1000 PC104 Camera \\
Card \\
(Additional Performance Tests) \\
SI PC104 Working Group \\
September 13, 2005
\end{tabular}

Test Plan Review Topics
Test Overview and Goals
Test Approach and System Configuration
Hardware and Software Components
Performance Test Plan
Pesources and Test Schedule
Spptriber 12,0005

Test Overview and Goals

- SI PC104 System Performance Tests

- Validate performance of a fully modified (per recommendation) PC104 SI camera card

Kantron CPU test NOT APPLICABLE

for $\$$ PI PC104

- Verify operation of Enhanced Write Filter (EWF) on LEC PC104 system

- Diagnose power cycle and initialization problems on SI PC104 system and provide recommendation for proper procedure

\section{Test Approach}

- Configure a SI PC104 stack with modified SI camera card

- Use a SI Simulator for testing with modified camera card tests

- Use S1-1000 camera for EWF and power cyde tests.

- Use the SI PC104 Test stand with Diamond I/C card to monitor buss vol tages.

- Use PC Test-station with UEC Test program to drive system through image aoquisition cydes.

Tests to be performed in B391 Room B350

Septrmber 12,2005

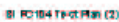

npenter 10,200 
SI PC104 Performance Test Report

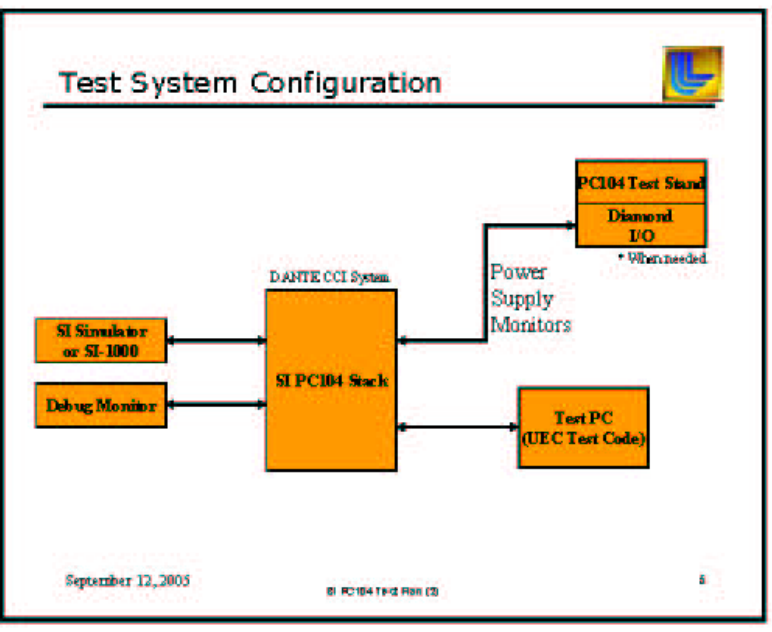

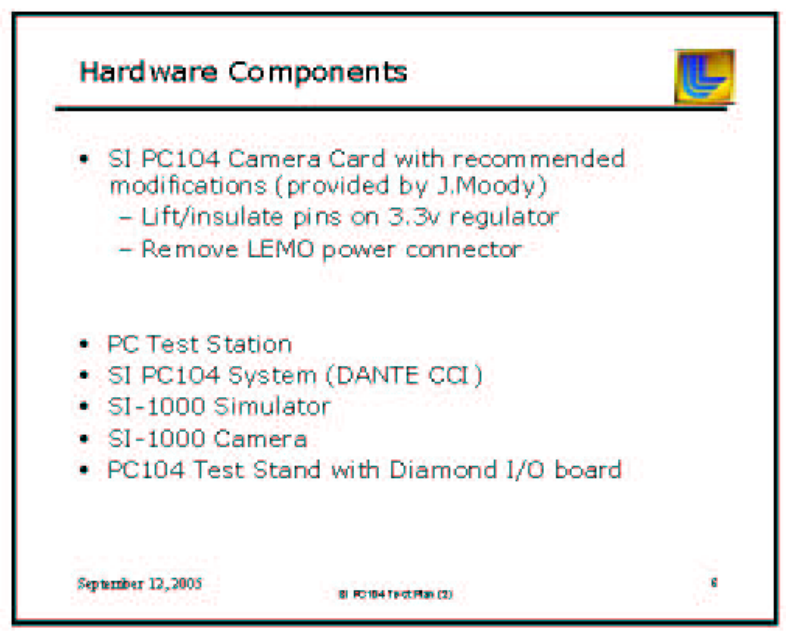

\section{Software Components}

- LUEC Test Program

- Diamond $1 / 0$ data capture software

- DOC with current released UEC code (V1.1.6)

- Configured with Enhanced Write Filter in ON state

- Read Exposure issue will cause image acquisition failure $\sim 10$ cydes.

- To be fixed in next release

- Modified UEC Java Driver for SI Simulator

- Driver takes into acoount $\$ 1$ Simulator timeout due to not having real $C \mathrm{CD}$.

- Reload driver after every reboot of SI PC104

Sequeniber 12,2005

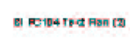
,
Performance Test Plan - SI Camera Card

- Purpose

- Validate performance of a fully modified PC1045] camera card.

- Test Plan

- Run continuous image acquisition cycles over a $\sim 2$ day period

- Monitor SI PC104 system performance

- Monitor/record SI PC104 system buss vol tages

$\left(+3.3 V_{,}+5 V_{,}+12 V\right)$

- Record failures/issues

- Evaluate results

September 12,2005 B) 


\section{SI PC104 Performance Test Report}
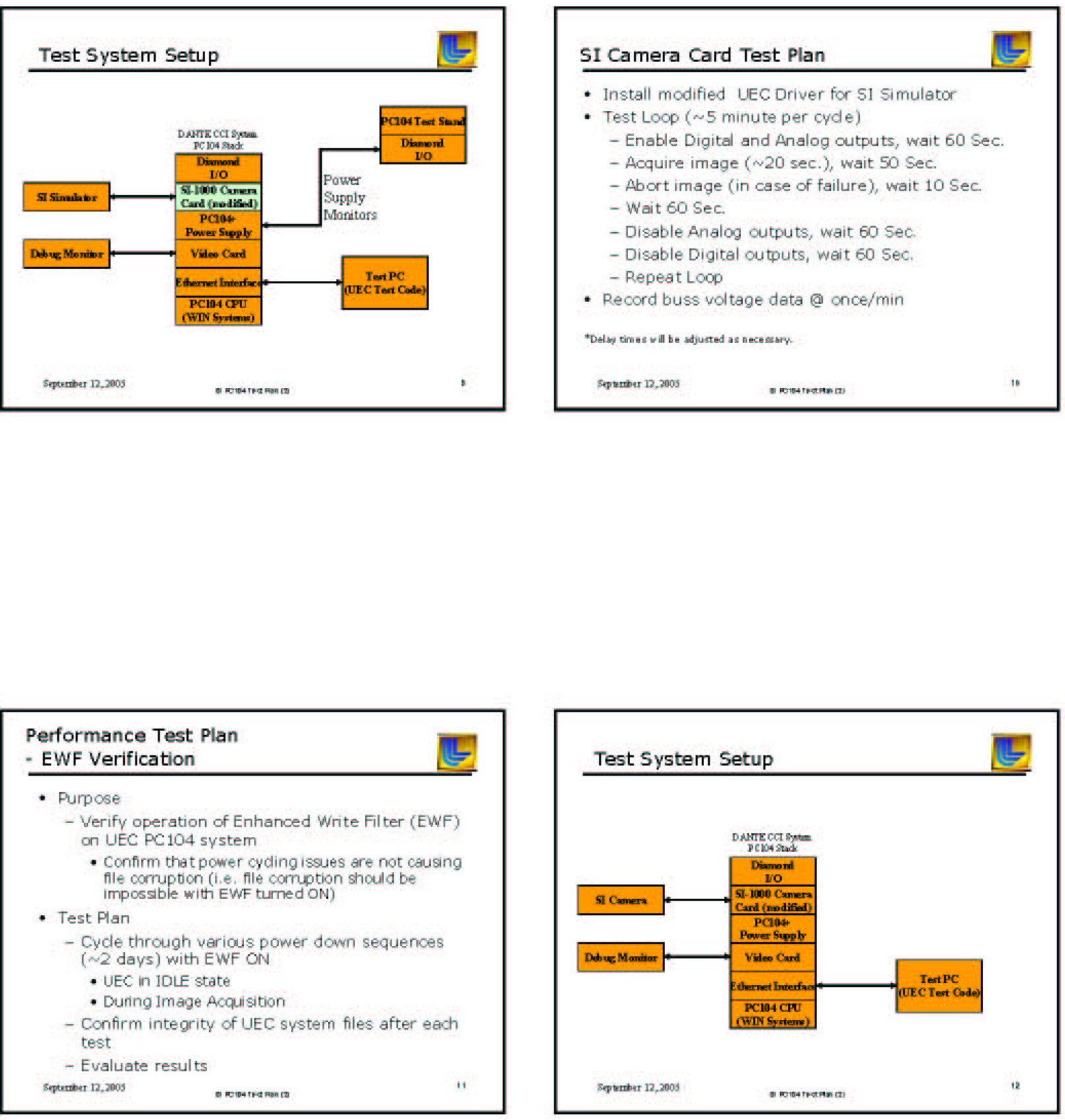
SI PC104 Performance Test Report

\section{EWF Verification Test Plan}

- Starting point for each test is with DC and UEC systems ON with communication working (i.e. able to take image)

- If communic ation is down

+ Close the UecTest program (Disconnects DC from UEC)

- Use teinet to log onto UEC system

+ Run the "shutdown. cmi" script to shut down the system

+ Cut power to UEC sy stem and camera; restore after $10 \mathrm{sec}$

- Open UecTest program, and verify communication by acquiring an im age

- Copy initial set of files to Test PC prior to starting test

- UEC System File Verification

- Mount Cildrive on the UEC computer

- Use "fe" command to compare files with initial set

- Create script to compare individual files

- Create log file for each comparison

\section{- UEC System Files}

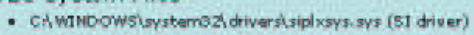

- CINIMDOWWSisystemozi ker nel32.dII

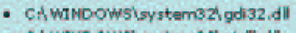

- CA WIND-OWSTs gsteme2/ ntall d

- Ci GXDISITASJN IInterface di

- ChGxDltasutility dil

- cilexbluecijar

September 12,2005

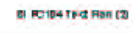

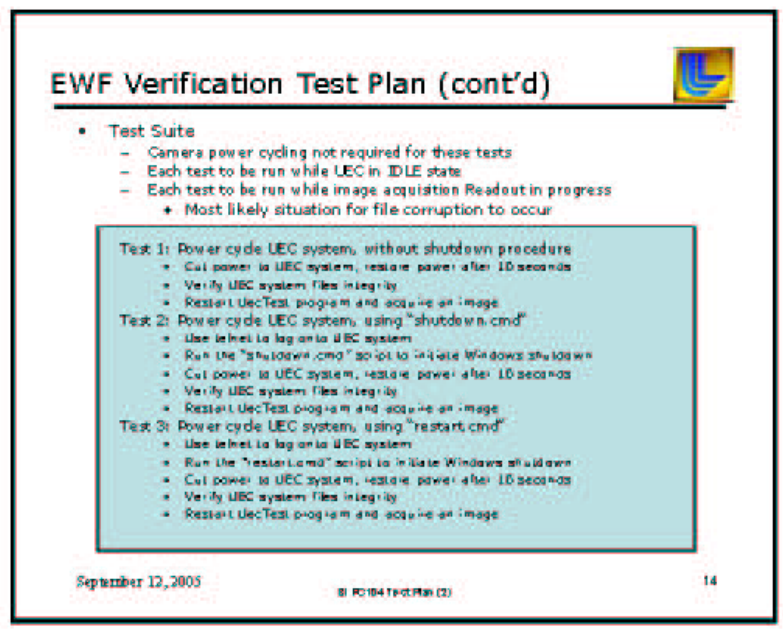

Performance Test Plan

- Power Cycle

- Purpose

- Diagnose power cyde and initialization problems on 81 PC104 system and provide recommendation for proper procedure

- Test Plan

- Perform various power cycle sequences of SI PC104 system ( $\sim 2$ days)

- Uec in jole state

- During Image Acquisition

- Establish success and failure for each sequence

- Evaluate results

- Recommend power cyde process for the SI PC104 system

September 12,2005

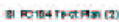


SI PC104 Performance Test Report
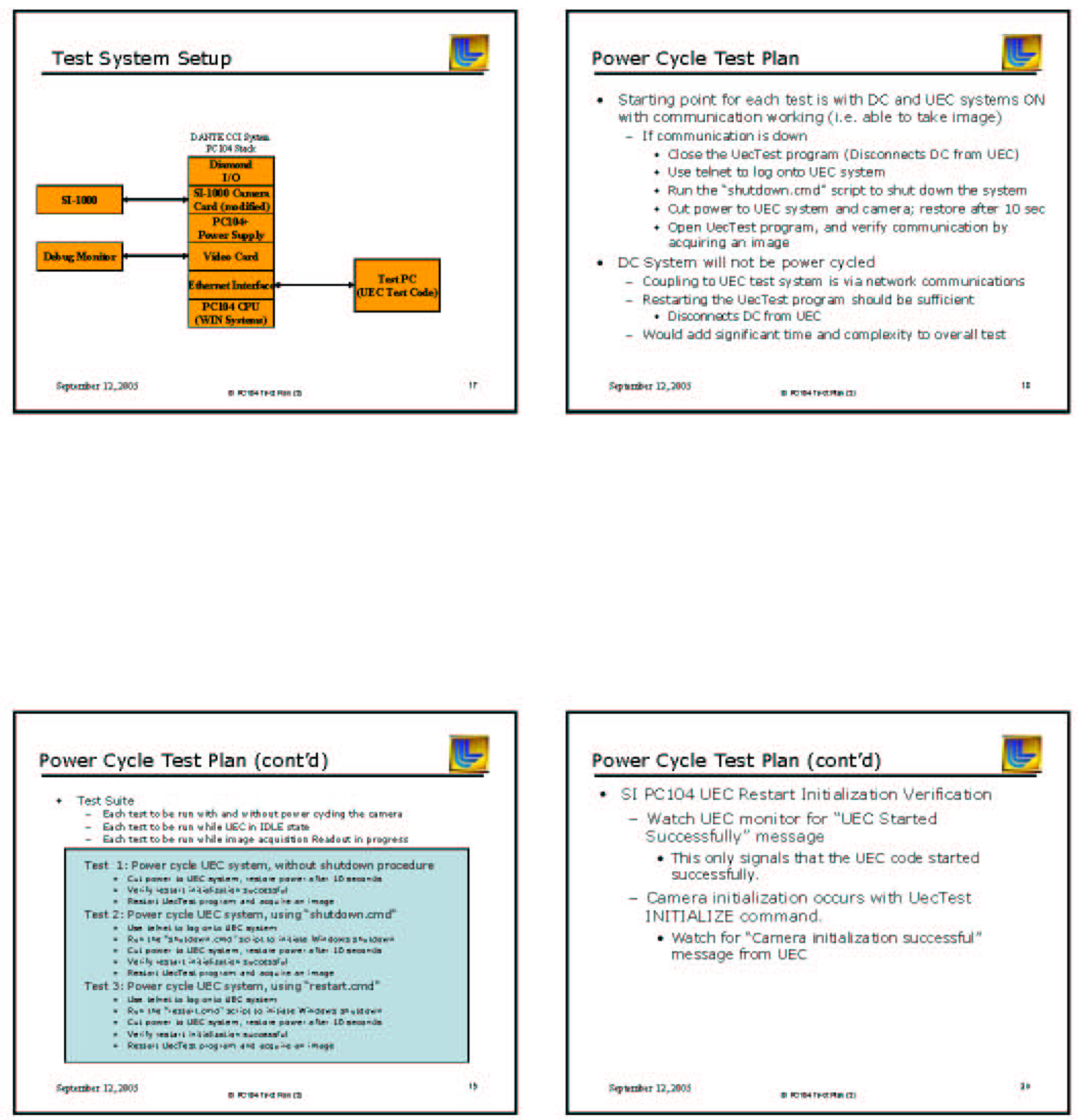


\section{SI PC104 Performance Test Report}

\section{Performance Test Expectations}

- No image acquisition failures with modified camera card

- SI PC104 buss voltages stay within specs

- Power cyde of S1 PC104 does not cause UEC system file corruption with DOC. Enhanced Write Filter turned ON

- Proper power cycle process defined as to not cause initialization problems on restart

Test Schedule

- B391 Test System Setup

- September 12-13

+ SI PC104 Camera Card Mods: J Moody

+ DANTE CCI system: K.Piston

- UEC Test PC: J.Nelson

- Performance Tests

- September 14-23

- Evaluate Test Results

- September 21-23

- Present Results

- Week of September 26 S.Montelongo 


\section{SI PC104 Performance Test Report}

\subsection{Appendix D: Test Plan 2 (Results for the SI-1000 PC104 Camera Card)}

Test Plan 2 Results were presented to the PC104 Working Group Team on November 17, 2005. The presentation included

Test Overview/Goals

Test Implementation and System Configuration

Hardware and Software Components

Test Results

Test Plan 2 culminated with a second round of testing.

The first round of testing showed that the PC104+ power supply was inappropriate for the SI PC104 system as it supplied +3.3vdc to the PC104 buss as did the SI PC104 camera card which also has an onboard +3.3vdc regulator. Spectral Instruments does not recommend having both a PC104+ power supply and the SI camera card regulator both provide the $+3.3 \mathrm{vdc}$ as a long term solution. It was also found that the PC104+ power supply $+3.3 \mathrm{vdc}$ rise time was inadequate stand-alone to meet the SI PC104 system timing requirements for SI driver installation.

A PC104 format power supply providing only $+5 \mathrm{vdc}$ and +12 vdc was recommended and installed in the SI PC104 system. This allowed the SI camera card to provide the $+3.3 \mathrm{vdc}$ buss voltage. Round 2 of testing validated the performance $(+3.3 \mathrm{vdc}$ rise time, SI driver installation) of the PC104 power supply configured into the SI PC104 system. 


\section{SI PC104 Performance Test Report}

\section{SI-1000 PC104 System}

Performance Test Results

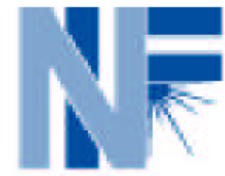

SI PC104 Working Group November 17, 2005

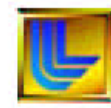

Personnel Involved with Test

- Jim Moody

- Sam Montelongo

- Jarom Nelson

- Ken Piston

- Joe Holder

- Joe Kimbrough
Test Plan Review Topics

$>$ Test Overview/Goals

$>$ Test Implementation and System Configuration

> Hardware and Software Components

> Test Results

\section{Test Overview and Goals}

- SI PC104 System Performance Tests - Validate performance of a fully modified (per recommendation) PC104 SI camera card.

- Test SITA.SJNInterface.DLL dated August 9,2005.

- Fixes the Exposure/Read issue causing abort of im age acquisition.

$$
\text { NOT APPLICABL.E for ST PCID4 }
$$

- Verify operation of Enhanced Write Filter (EWF) on LEC PC104 system

- Diagnose power cycle and initialization problems on SI PC104 system and provide recommendation for proper procedure 
SI PC104 Performance Test Report

\section{Test Approach}

- Configure a SI PC104 stack with modified SI camera card

- Monitor PC104 buss voltages via PC104 Test station - $+3.3 v_{,}+5 v,+/-12 v$

- Use a SI Simulator for testing with modified camera card tests.

- Use SI-1000 camera for EWF and power cycle tests.

- Use PC Test-station with UEC Test program to drive system through image acquisition cycles.

- Use DANTE CCI as the test system

- Most extreme configuration for PC104 diagnostic stack

\section{Test Implementation}

- Test Dates: 9/12/05 - 11/11/05

- Test Location:B391 Rm B350

- Test System

- DANTE CCI with SI-1000 Simulator

- SI CCD Camera 1000-109, 1000-106

- PC104 Test Stand

- Modified SI Camera cards: $-107,-104$

PC104 Power Supply HE104+DX

- SI PC104/DOC running UEC Ver. 1.1 .6

- SI PC104/DOC SI Driver: SIPLXSYS.SYS ReV. A

- SITASJNIInterface.DLL (versions dated 6/22/04 \& 8/9/05)

- DSP initialization file for SI-1000, 3628D.bin

- UECTestC code

- Camera initialization

- Image acquisition cycle: single or continuous
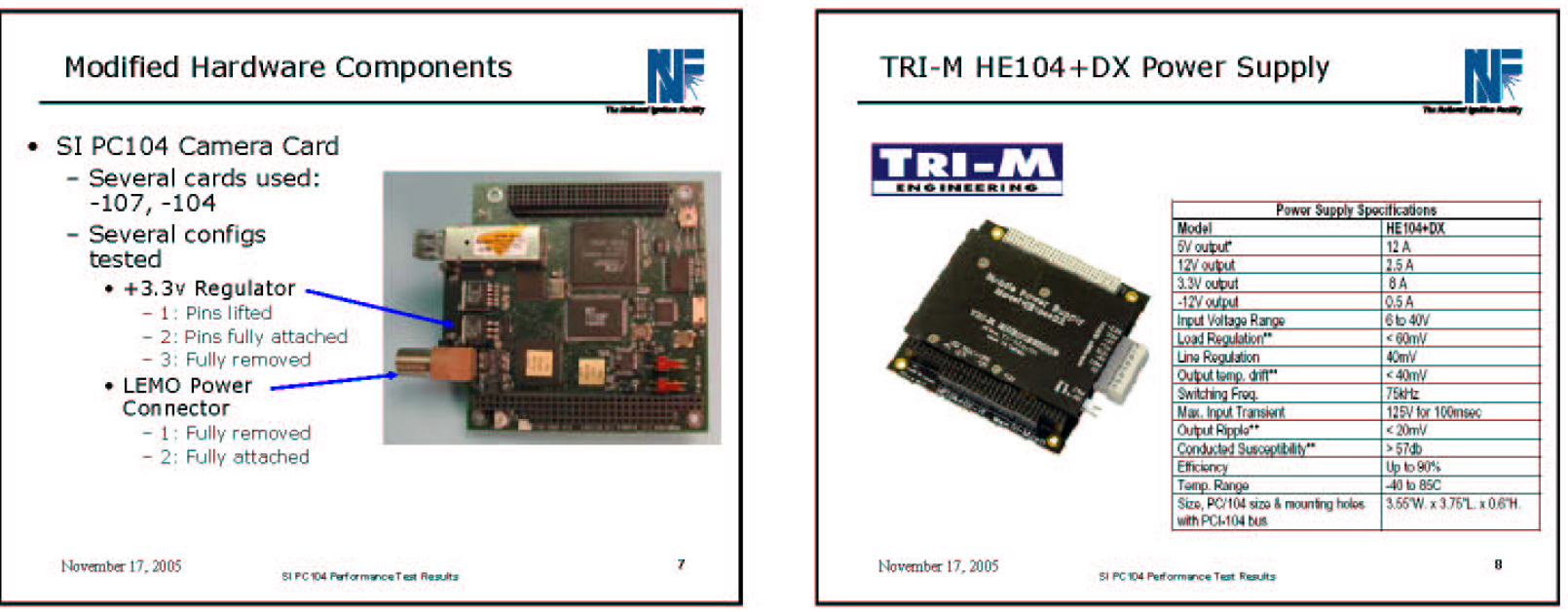


\section{SI PC104 Performance Test Report}
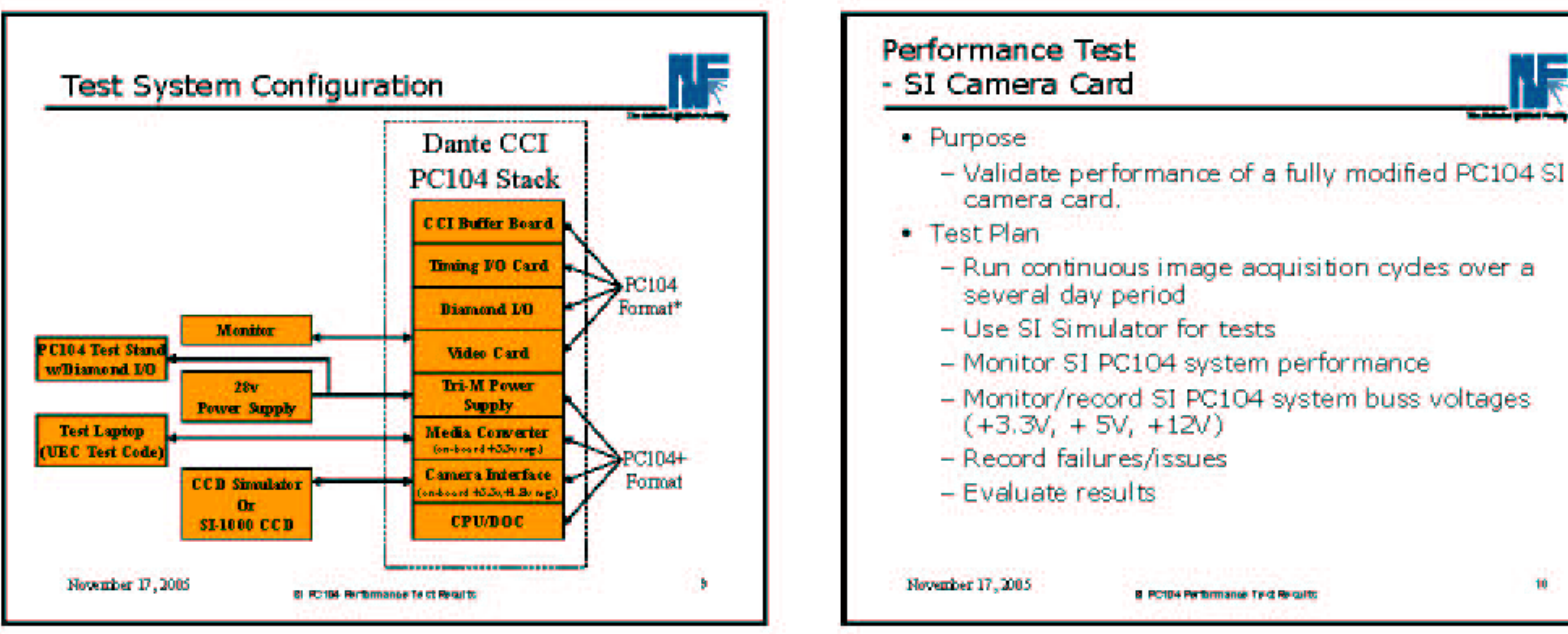

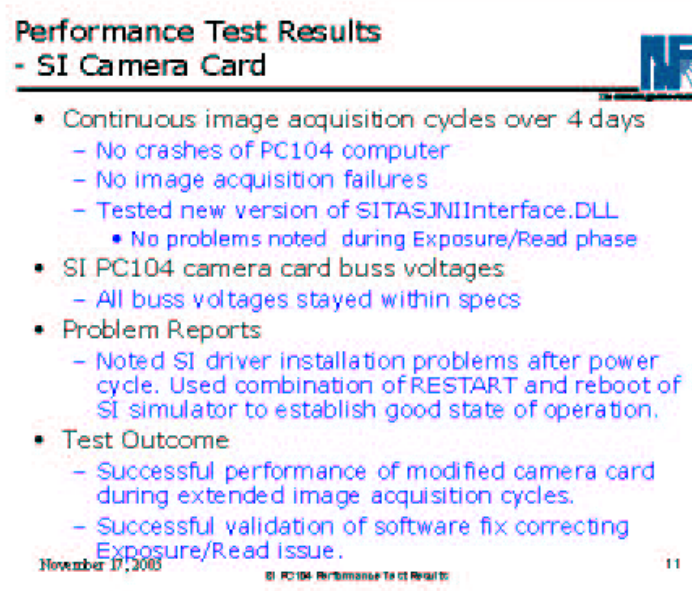

- Continuous image aoquisition cydes over 4 days

- No crashes of PC104 computer

- No image acquisition failures

- Tested new version of SITASINIInterface.DL - No problems noted during Exposure/Read phase

- SI PC104 camera card buss voltages

- All buss voltages stayed within specs

- Problem Reports

- Noted SI driver installation problems after power cycle. Used combination of RESTART and reboot of SI simulator to establish good state of operation.

- Test Outcome

- Successful performance of modified camera card during extended image acquisition cycles.

- Successful validation of software fix correcting

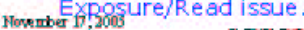

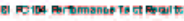

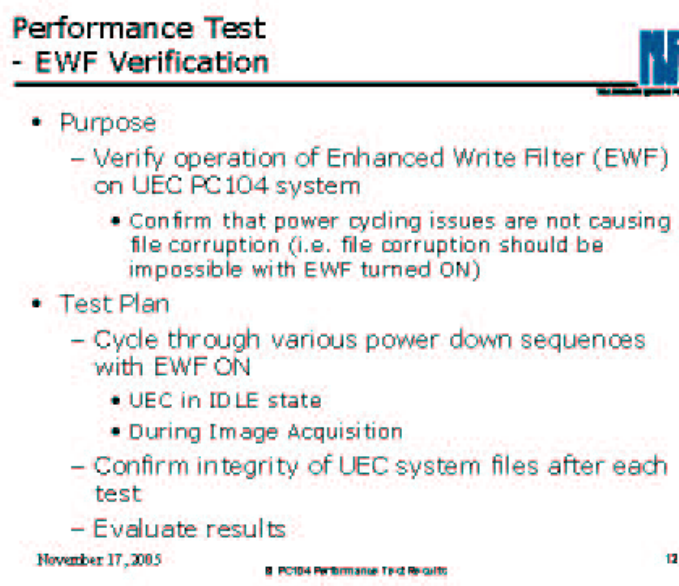


SI PC104 Performance Test Report

Performance Test Results

- EWF Verification

- Problem Reports

- Noted SI driver installation problems after power cucle. Used combination of RESTART and reboot of SI simulator to establish good state of operation.

- Test Outcome

- Numerous power cycles (28v cutoff, Shutdown,

Restart) under various conditions of $\mathrm{PC1} 104$ System (Idle, Image Acquisition READ) did not cause file corruption.

- System file integrity successfully verifled after each test.

Performance Test Results

- Power Cycle

- Problem Reports

- SI Driver Installation

- S1 driver will not install with Camera Card +3.3vdc

regulator rem oved or pins detached, Rise time

inadequate $(\sim 125 \mathrm{msec})$.

- SI states 50 msec minimum.

+ SI driver succesful installation with Camera Card +3.3vdc

regulator fully attached. Rise time adequate ( a 5 masec).

- Soft RESTART successful but.. SI camera PWR LED failure

- Soft RESTART successful but.. SI cam
indication upon initial communication.

- Recommendations

- SI: Having the +3.3vdc regulator fully attached while using

the HE $104+$ DX power supply to also provide $+3.3 y d c$ will

cause early component failure.

PC104WG: Install a PC104 form at Power Supply that provides

only $+5 \mathrm{vdc}$ and $+12 \mathrm{vdc}$. Full attach $+3.3 \mathrm{v}$ regulator.

- Test Outcome

- Pursue PC104 form at Power Supply - Tri-M HE104-75W

Howermer 17,2005

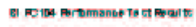

Performance Test

- Power Cycle

- Purpose

- Diagnose power cyde and initialization problems on 51 PC 104 system and provide recommendation for proper procedure

- Testplan

- Perform various power cycle sequences of S1 PC104 system

- Uec in ID Le state

- During Image Acquisition

- Establish success and failure for each sequence

- Evaluate results

- Recomm end power cycle process for the SI PC 104 system

PC104 +3.3vdc signal measured at power up using HE104+DX Power Supply

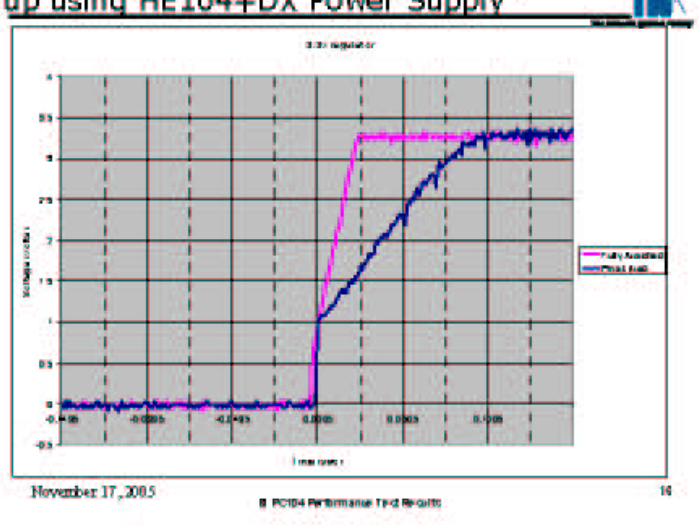


SI PC104 Performance Test Report
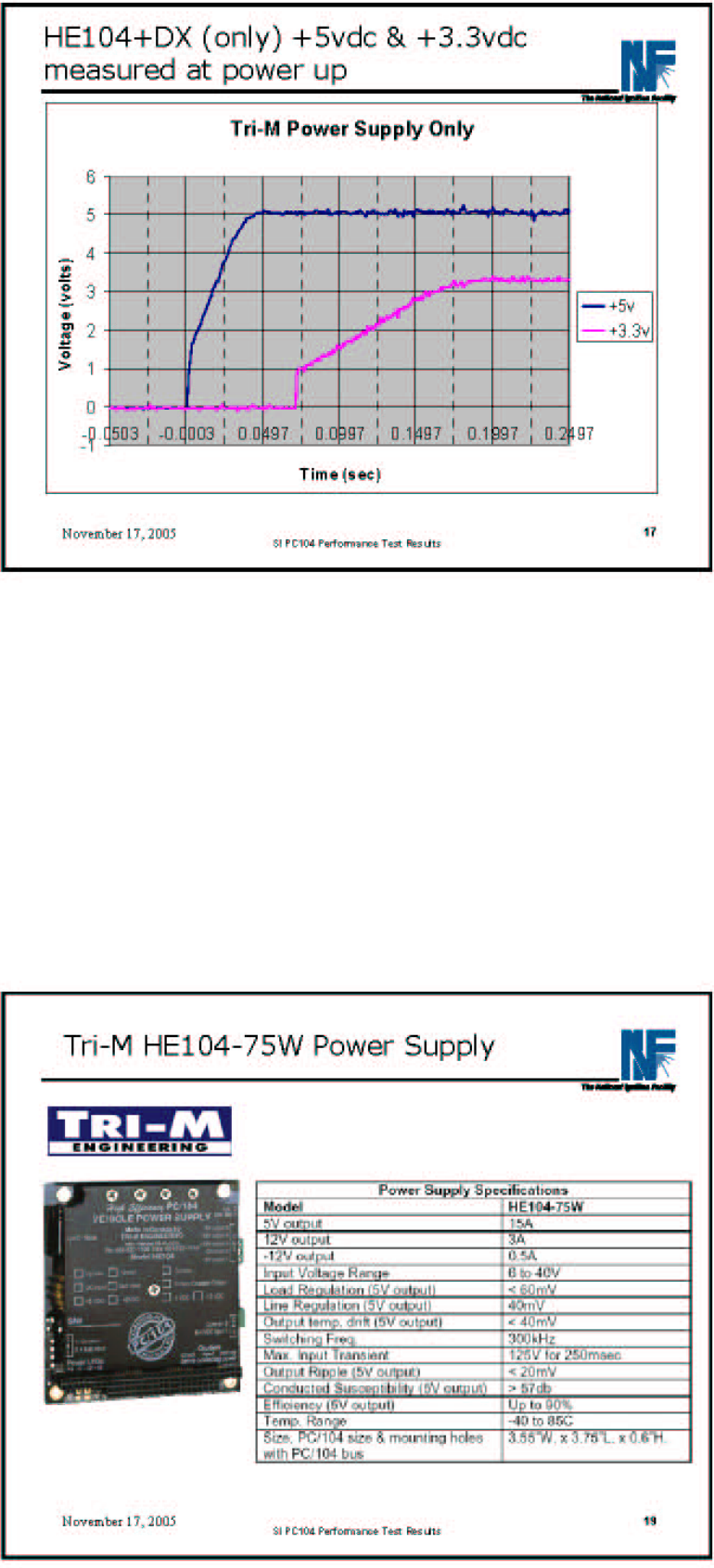
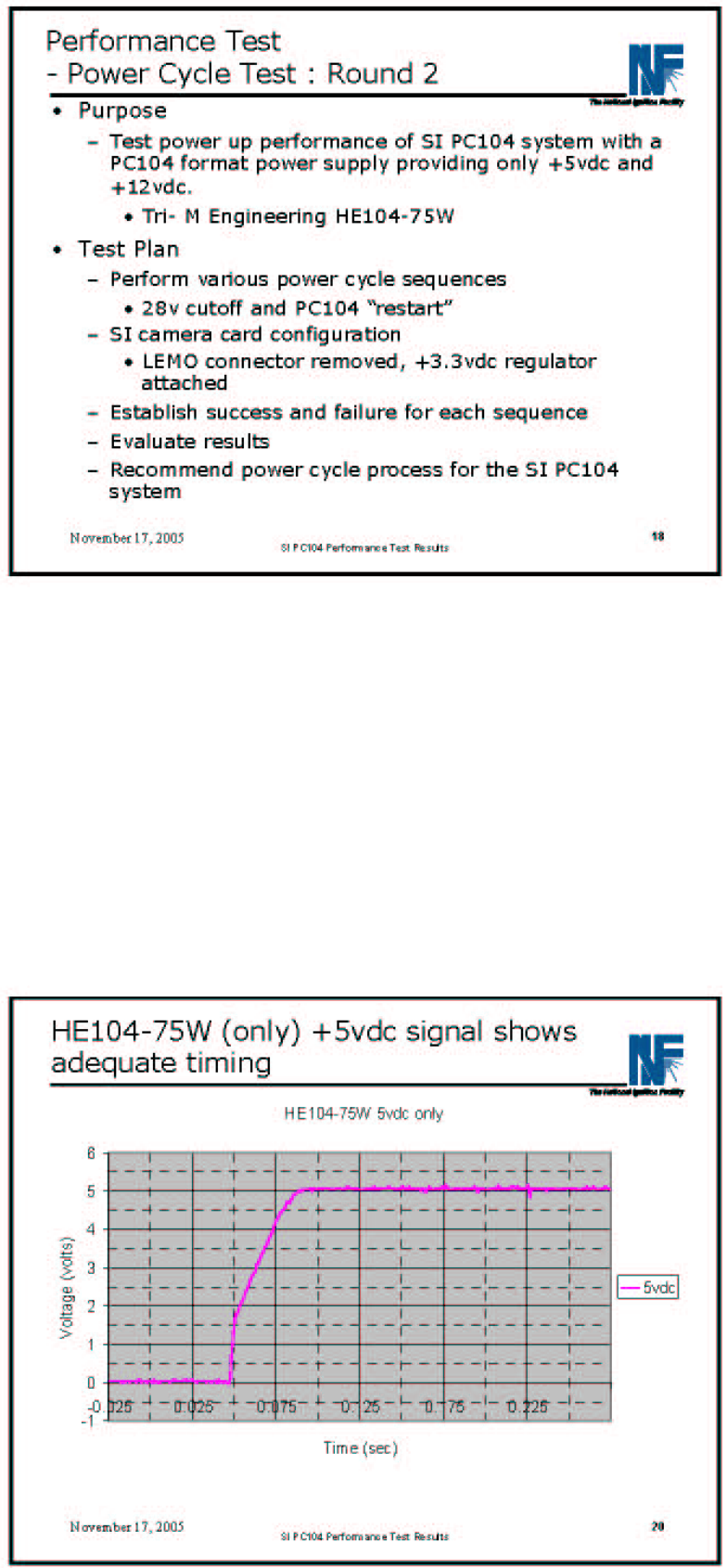
SI PC104 Performance Test Report

PC104/HE104-75W $+3.3 v$ and $+5 v$ signals measured at power up

PC 104 Euss Voltages using HE104750

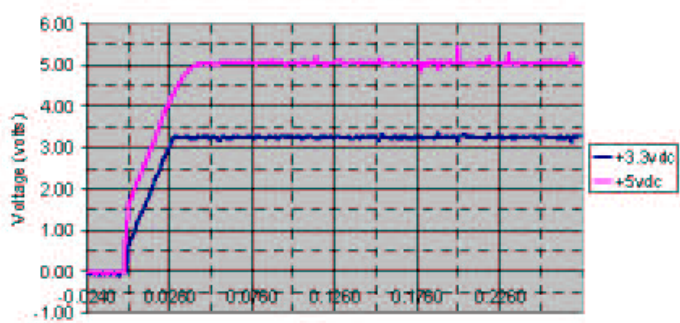

Time (sec)

Performance Test Results

- Power Cycle Test : Round 2

- Problem Reports

- PC104 operating sy stem "restart" although successiul in reboot caused camera communications to fail (inconsistent) upon initial communication to CCD.

+ SI response: Update SI PC104 components to latest rev levels.

- Test Outcome

- SI driver install successful after every full 28v power cycle.

- LEMO connector detached did not change performance.

- Camera card +3.3vdc/ +5vdc rise time measured to be adequate per SI requirement ( $550 \mathrm{msec}$ )

- Recommendation

- Use "restart" comm and only for test and debug. Not recommended for use during operations.

- Always use a full 28vdc power cycle to cleanly reboot PC104
PC104/HE104-75W: Buss voltages within specs during image acquisition loop test.
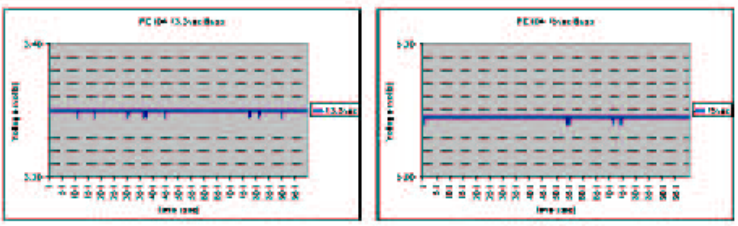

- All data taken at 1 sec interval

+1000 secs representative of $\sim 4$

hour image acquisition loop

Noveriber 17 , 2005

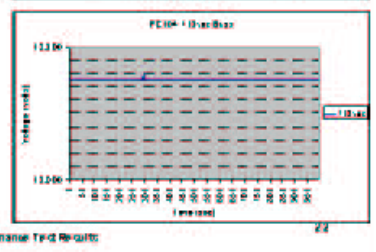

SI PC104 Performance Test Results Summary

- SI Camera Card

- Successful performance of modified camera card during continuous i mage acquisition cycles.

- Successful data acquisition during Exposure/Read phase. No ABORTs noted.

- SITASINIINterface dll dated $8 / 9 / 05$

- DOC. EWF File Verification

- Successful system file integrity verification after multiple and various methods of power down.

- Power Cyde Reliability

- Consistent successful system power up (SI driver installation) after everv full $28 \mathrm{vdc}$ power cucle.

We now have a SI PC104 system that reliably powers up every time upon a full $28 v$ power down.

Nowember 1T, Dos

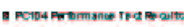




\section{SI PC104 Performance Test Report}

\section{SI PC104 Recommendations}

- Update LLNL "SI Camera Usage Recommendations Memo" and send out to SI user group.

- Inform Spectral Instruments of test results

- Use of the Tri-M Engineering HE104-75W power supply.

- Send all SI 1000 cameras and PC10 4 systems to SI for update to latest revision levels (hardware and software). - J.Moody to schedule

- Run LLNL performance tests with latest hardware and software available from both $S I$ and $\amalg N L$.

- Include im age acquisition perform ance loop test as part of LLNL system verification prior to release of upda ted systems

- Update existing SI PC104 systems with latest

SITASINIInterface.dll.

- Information subm itted to J.K amperschroer for entry into SCR Track.

Noveriber 17,2006

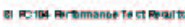

Schedule

- Test System Configuration and Setup : June 13-27, 2005

- Performance Test I : June 27-July 6, 2005

- Present Test Results i July 15, 2005

- SI PC104 Usage Recomm endation Memo: August 23, 2005

- Performance Test II : September 12 - November 11, 2005

- SI PC104 Usage Recommendation Memo Update: Nov. 30, 2005

- 51 PC104 Perform ance Test Report capturing tests, results and recomm endations

- November 30, 2005 S.Montelongo/D. Nelson

- Implement Stress Tests: Schedule TBD by new Lead

Howember 17,2005

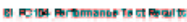

Recommended Stress Tests to Pursue

- Raise ambient temperature of SI PC104 System to $40^{\circ}+20 \%$ (SXI boundary).

- Vary SI PC104 System Input Power (28V) to vendor specified boundaries.

- Vary SI-1000 Camera Power Supply (28V) to vendor specified boundaries.

- SI PC104 System Vibration

- Contact when ready

- Tom Woehrle, WTG, X3-1716

Verify image quality pre/post each test.

Noveriber 17, 2005

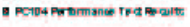




\section{SI PC104 Performance Test Report}

\subsection{Appendix E: Memo \#1 (SI PC104 Usage Recommendation Memo)}

The SI PC104 Usage Recommendation memo was reviewed with the PC104 Working Group Team prior to sending out to the SI PC104 user community. Recommendations were discussed and reviewed with Spectral Instruments prior to completion. 


\section{SI PC104 Performance Test Report}

Interdepartmental

Mail Station $L-459$

Ext: $\quad 4-9824$
NATIONAL IGNITION FACILITY

PROGRAMS DIRECTORATE

December 13, 2005

TO: Spectral Instruments PC104 Users

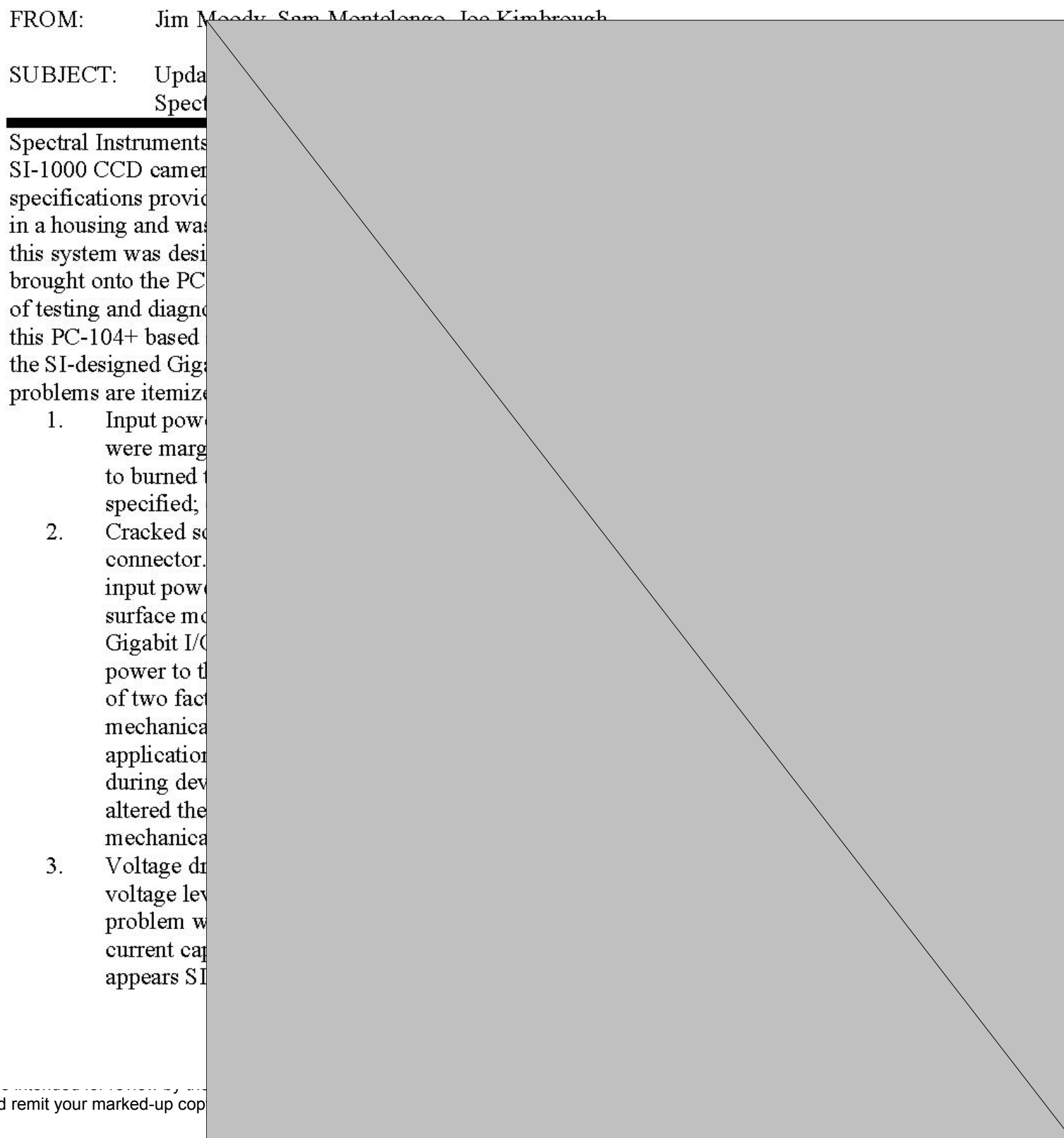




\section{SI PC104 Performance Test Report}




\section{SI PC104 Performance Test Report}

The solution for these problems was to add a Tri-M Engineering HE104-75W Power Supply module into the SI PC-104 stack. This board requires the NIF standard $+28 \mathrm{~V}$ input and provides the $+5 \mathrm{~V}$ and $\pm 12 \mathrm{~V}$ supplies for the $\mathrm{PC}-104$ systems. There is a $+3.3 \mathrm{~V}$ regulator on the SI Gigabit $\mathrm{I} / \mathrm{O}$ card that provides the $+3.3 \mathrm{~V}$ to the SI cards. This voltage is tied to the $\mathrm{PC}-104+$ bus and could possibly provide $+3.3 \mathrm{~V}$ for future card upgrades. However, if that is to be done, it is recommended that a power analysis of the $+3.3 \mathrm{~V}$ for the system and the proposed card be completed. There is also an additional $+1.8 \mathrm{~V}$ regulator on the SI Gigabit I/O card that provides power only to an FPGA; this regulator has not had any problems and will remain. The advantages of using this board are:

1. Eliminates power input through connector on SI Gigabit I/O card.

2. Eliminates requirement of the SMT filters (the components that have had problems with cracked solder joints).

3. Eliminates concerns with undersized input power traces on SI Gigabit I/O card.

4. Provides increased current capacity for all voltages required by the system.

This implementation was tested in November 2005 for an extended period of time. No power related failures were recorded.

The recommendations of the test team for all SI PC-104+ configurations (especially those implementing the SI PC-104+ outside its originally intended configuration) are:

1. Do not use the Lemo power connector from the SI Gigabit I/O card. If required in tight designs, this connector can be removed. However, it is not recommended, if avoidable.

2. Design diagnostics using the SI PC-104+ system to include the Tri-M Engineering HE104-75W Power Supply module. This board supplies power to all PC-104 busses.

a. Alternate method: Provide an external power supply capable of producing adequate power to busses via the PC-104+ buss connectors. Do not use the LEMO connector. Include a power requirements discussion as part of the diagnostics design review.

3. The SI power supply will no longer provide $+24 \mathrm{~V}$ to the two fans used to cool the $\mathrm{PC}-104+$ module. Fans will need to be implemented in one of the following ways:

a. Preferred method: Implement $+12 \mathrm{~V}$ fans .

b. Alternate method: Connect the +12 and $-12 \mathrm{~V}$ supplies differentially to the fans.

c. Alternate method: The diagnostic designer can provide their own fan system.

4. Ensure all PC-104 and PC-104+ cards intended for use conform to the PC-104 or $\mathrm{PC} 104+$ specification.

cc. Dennis O’Brien

Brian MacGowan 


\section{SI PC104 Performance Test Report}




\section{SI PC104 Performance Test Report}

\subsection{Appendix F: Memo \#2 (SI PC104 Usage Recommendation Update Memo)}

The SI PC104 Usage Recommendation Update memo is based on results obtained from Test Plan 2. When complete it will be forwarded to the SI PC104 User community and Spectral Instruments. The document will be located on the NIF Server under the PC104 folder.

The update to initial recommendations will be based on the following:

Issues found during further tests showed problems with the SI driver installation upon bootup. It would not consistently install after power up. This problem was noted in prior tests and further evaluation was required to trace down the root of the problem.

The root of the problem was due to the SI PC104 CPU buss voltage timing requirements not being met with the selected PC104+ power supply module (HE104+DX) for the $+3.3 \mathrm{vdc}$ line. The power supply $+3.3 \mathrm{vdc}$ signal rise time was too slow $(\sim 125 \mathrm{msec})$. Spectral Instruments requires a rise time of $50 \mathrm{msec}$ or less for proper driver installation.

The SI driver installation problem did not exist if the SI PC104 camera card $+3.3 v d c$ regulator was fully attached and the PC104+ power supply was used. In this case both the PC104+ power supply and the $+3.3 \mathrm{vdc}$ onboard regulator (SI PC104 camera card) were generating +3.3vdc. Spectral Instruments did not recommend this as a long term solution.

The solution was to replace the PC104+ power supply with a PC104 power supply (HE104-75W) and to leave the SI camera card +3.3vdc regulator fully attached. The PC104 power supply only generates $+5 \mathrm{vdc}$ and $+/-12$ vdc allowing the SI camera card to provide the $+3.3 \mathrm{vdc}$ signal required by the SI PC104 system. 


\section{SI PC104 Performance Test Report}

\subsection{Appendix G: PC104 Buss Voltage Waveforms}

As stated by Spectral Instruments in the (edited) email below, the +3.3vdc signal must have a rise time of $50 \mathrm{msec}$ or less.

From: "Roger W. Cover" <rcover@specinst.com>

To: "Jim Moody" < moody8@1lnl.gov>

Cc: "Sam Montelongo" < montelongo1@1lnl.gov>,

"Hannes Meyer"<hmeyer@specinst.com>, "Gary Sims" <gsims@specinst.com>

Subject: PC-104+ Power Supply Startup Timing

Date: Wed, 26 Oct 2005 08:57:11 -0700

Howdy Jim,

I have looked into the configuration timing I mentioned in my last email. Here are my results.

$<$ snip...>

While I was looking at the configuration timing, I discovered that the XC18V01 configuration PROM on the $\mathrm{PC}-104+$ camera interface board has a rise time requirement for its power supply. I have attached a snapshot of a portion of the datasheet for that part (XC18V01RiseTime.gif). It states that the $3.3 \mathrm{~V}$ supply needs to come up in $50 \mathrm{~ms}$ or less. This makes the power supply you are using unacceptable.

\begin{tabular}{|c|c|c|c|c|c|}
\hline \multicolumn{6}{|c|}{ Recommended Operating Conditions } \\
\hline Symbol & \multicolumn{2}{|l|}{ Parameter } & Min & Max & Units \\
\hline \multirow[t]{2}{*}{$\mathrm{V}_{\text {CCINT }}$} & Internal voltage supply $\left(\mathrm{T}_{\mathrm{A}}=0^{\circ} \mathrm{C}\right.$ to $\left.+70^{\circ} \mathrm{C}\right)$ & Commercial & 3.0 & 3.6 & V \\
\hline & Internal voltage supply $\left(T_{A}=-40^{\circ} \mathrm{C}\right.$ to $\left.+85^{\circ} \mathrm{C}\right)$ & Industrial & 3.0 & 3.6 & V \\
\hline \multirow[t]{2}{*}{$\mathrm{V}_{\mathrm{CCO}}$} & \multicolumn{2}{|l|}{ Supply voltage for output drivers for $3.3 \mathrm{~V}$ operation } & 3.0 & 3.6 & V \\
\hline & \multicolumn{2}{|l|}{ Supply voltage for output drivers for $2.5 \mathrm{~V}$ operation } & 2.3 & 2.7 & V \\
\hline $\mathrm{V}_{\mathrm{IL}}$ & \multicolumn{2}{|l|}{ Low-level input voltage } & 0 & 0.8 & $\mathrm{~V}$ \\
\hline $\mathrm{V}_{\mathrm{IH}}$ & \multicolumn{2}{|l|}{ High-level input voltage } & 2.0 & 5.5 & V \\
\hline $\mathrm{V}_{\mathrm{O}}$ & \multicolumn{2}{|l|}{ Output voltage } & 0 & $\mathrm{~V}_{\mathrm{CCO}}$ & $\mathrm{V}$ \\
\hline$T_{\vee C C}$ & \multicolumn{2}{|l|}{$V_{C C}$ rise time from $0 V$ to nominal voltage ${ }^{(1)}$} & 1 & 50 & $\mathrm{~ms}$ \\
\hline \multicolumn{6}{|c|}{$\begin{array}{l}\text { Notes: } \\
\text { 1. At power up, the device requires the } V_{C C} \text { power supply to monotonically rise from } 0 \mathrm{~V} \text { to nominal voltage within the specified } V_{C C} \text { rise } \\
\text { time. If the power supply cannot meet this requirement, then the device might not perform power-on-reset properly. }\end{array}$} \\
\hline
\end{tabular}

Typically the configuration time is not an issue. In this case it may become a non-issue with a different power supply. Let me know if there is any further information I need to provide.

Regards,

Roger W. Cover

Spectral Instruments, Inc.

420 N. Bonita Ave.

Tucson, AZ 85745

Voice: $520-884-8821$ ext. 144

FAX: 520-884-8803 


\section{SI PC104 Performance Test Report}

The following waveform shows the SI PC104 +3.3vdc buss measured at power on as a function of the SI camera card on-board regulator with pins lifted and pins fully attached. As can be seen by the waveform, with pins lifted the $+3.3 \mathrm{vdc}$ rise time is $\sim 125 \mathrm{msec}$, with pins fully attached the rise time is $\sim 50 \mathrm{msec}$.

$3.3 \mathrm{v}$ regulator

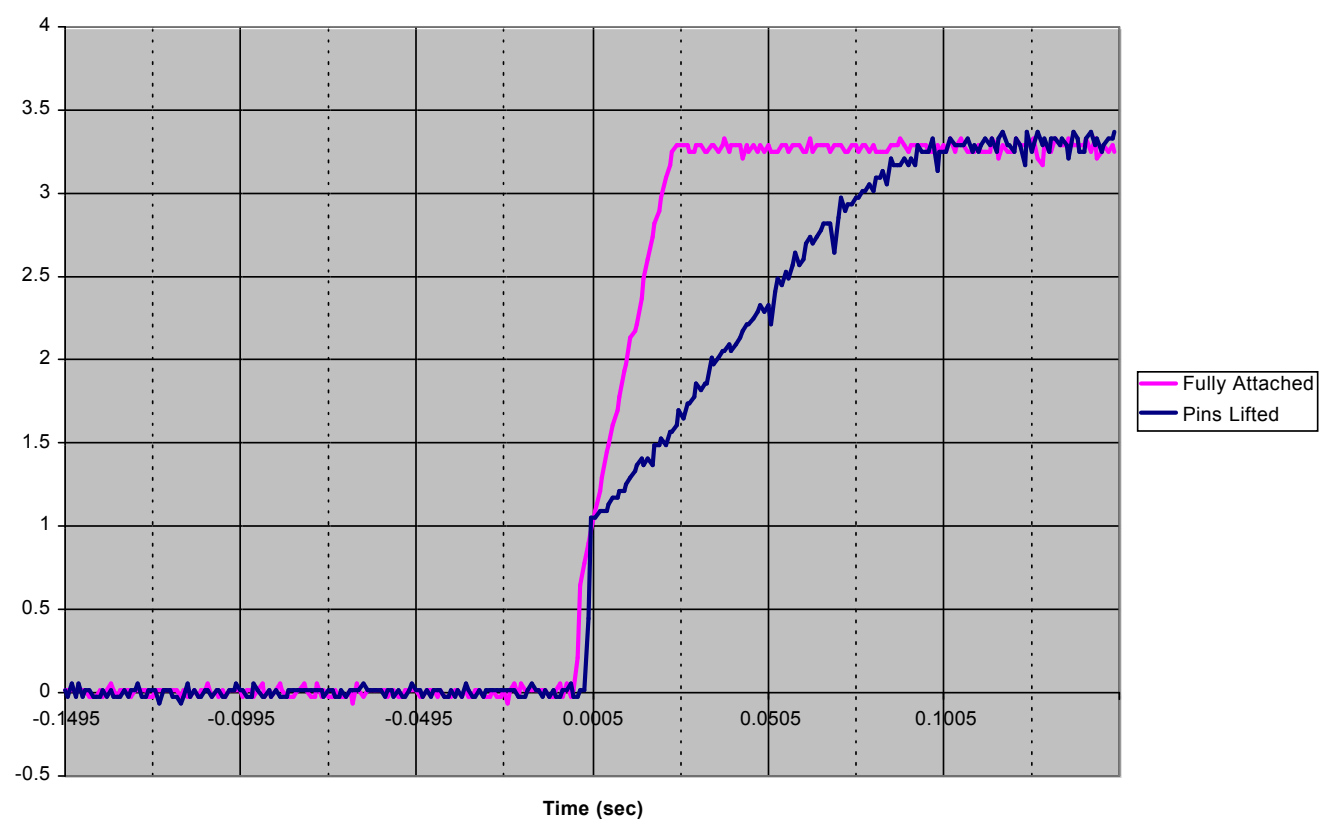




\section{SI PC104 Performance Test Report}

The following waveform shows the output of the (PC104+) HE104+DX $+3.3 \mathrm{vdc}$ and $+5 \mathrm{vdc}$ signals measured at power on. As can be seen by the waveform, the HE104+DX +3.3vdc rise time is $\sim 125 \mathrm{msec}$. This is too slow to meet SI power up requirements of $50 \mathrm{msec}$ or less.

\section{Tri-M Power Supply Only}

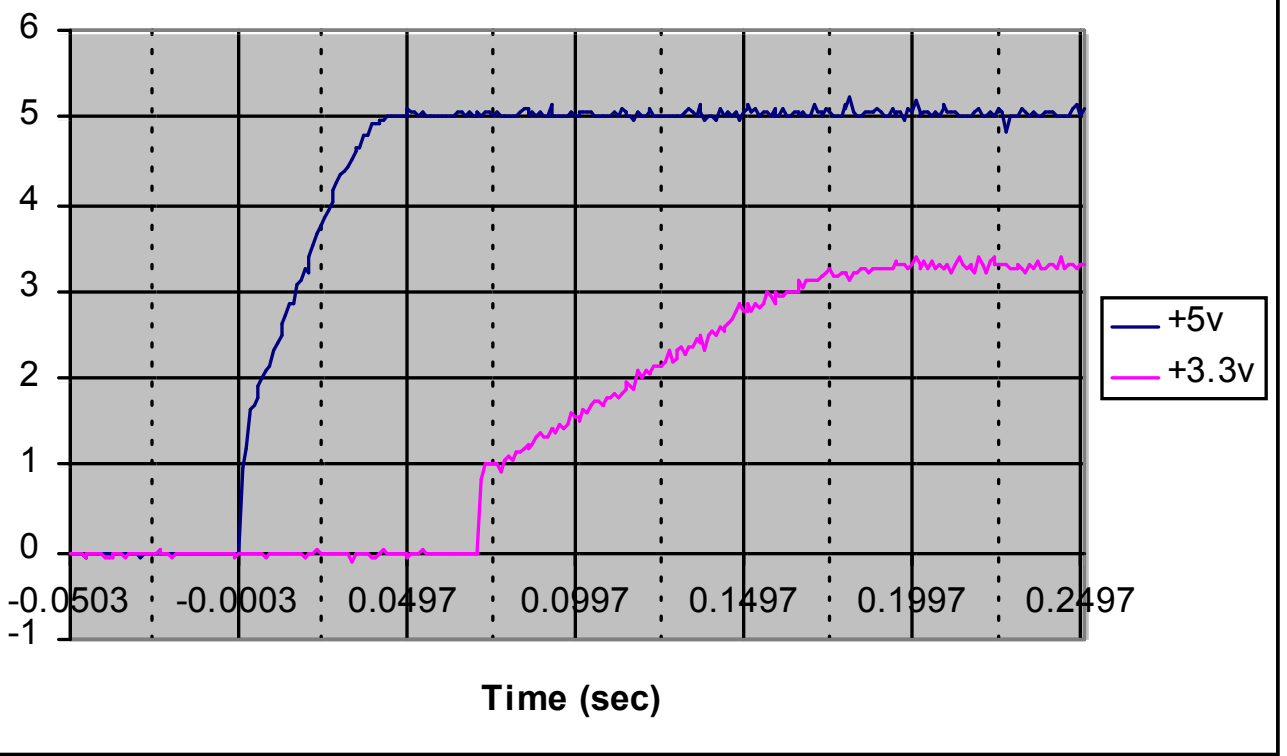




\section{SI PC104 Performance Test Report}

The following waveform shows the SI PC104 +3.3vdc and $+5 \mathrm{vdc}$ buss voltages using the HE104-75W measured at power on. As can be seen by the waveform, the $+3.3 \mathrm{vdc}$ rise time is $\sim 25 \mathrm{msec}$. This meets the SI power up requirements of $50 \mathrm{msec}$ or less.

PC104 Buss Voltages using HE104-75W

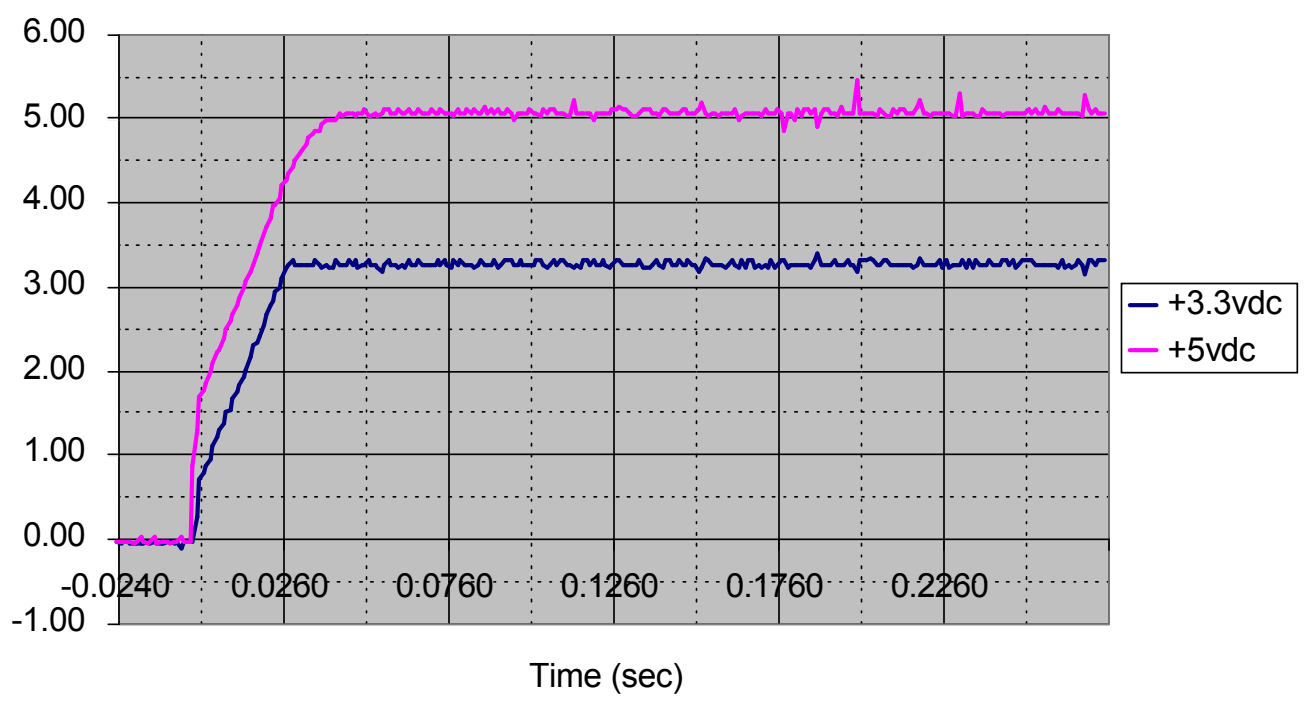




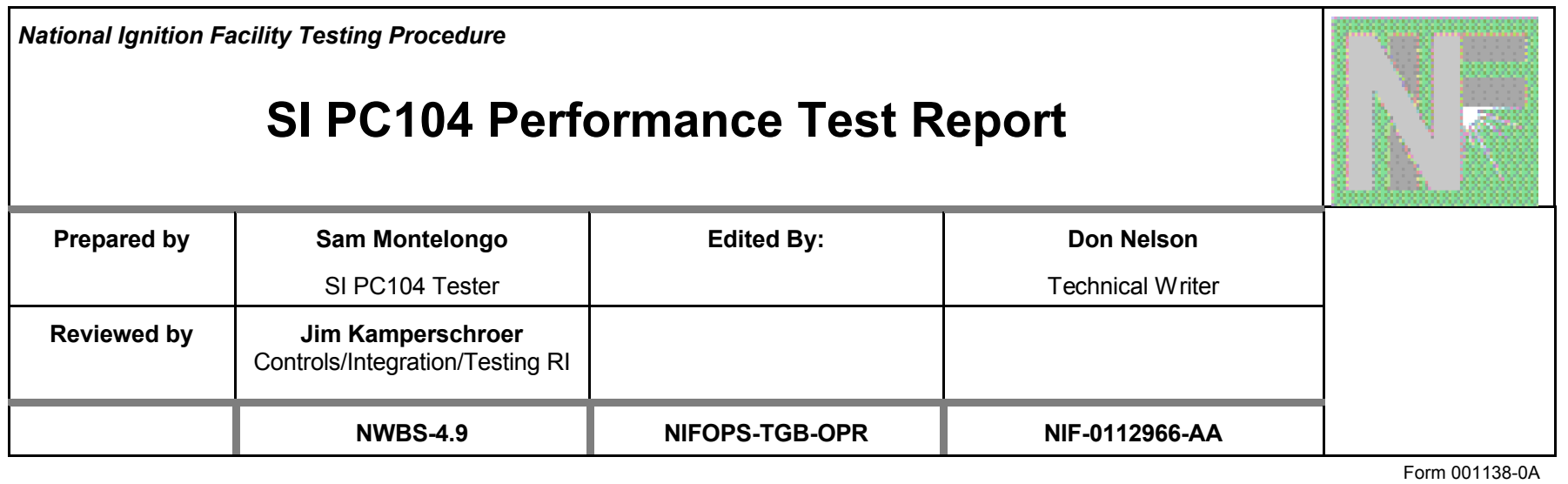

